ESAIM: COCV 27 (2021) 17

https://doi.org/10.1051/cocv/2021008
ESAIM: Control, Optimisation and Calculus of Variations

www.esaim-cocv.org

\title{
A PENALTY APPROACH TO THE INFINITE HORIZON LQR OPTIMAL CONTROL PROBLEM FOR THE LINEARIZED BOUSSINESQ SYSTEM
}

\author{
Kévin Le Balc'h and Marius TucsnaK*
}

\begin{abstract}
In this paper, we consider the infinite time horizon LQR optimal control problem for the linearized Boussinesq system. The goal is to justify the approximation by penalization of the free divergence condition in this context. We establish convergence results for optimal controls, optimal solutions and Riccati operators when the penalization parameter goes to zero. These results are obtained under two different assumptions. The first one treats the linearization around a sufficiently small stationary state and an arbitrary control operator (possibly of finite rank), while the second one does no longer require the smallness of the stationary state but requires to consider controls distributed in a subdomain and depending on the space variable.
\end{abstract}

Mathematics Subject Classification. 49N10, 93B05.

Received November 29, 2020. Accepted January 13, 2021.

Dedicated to Enrique Zuazua for his 60th birthday.

\section{INTRODUCTION}

The optimal control of the Boussinesq system and of its linearization around a stationary state are matters of great interest in various applications fields, such as designing and exploiting energy efficient buildings, (see, for instance, $[9,10,23,33])$. A difficulty which has to be handled in solving this type of problems is that, due to the free divergence condition for the velocity field and to the presence of the pressure, the governing equations cannot be written as a well-posed control system in the sense of Salomon-Weiss (as described, for instance, in Curtain and Weiss [12]), but merely as an infinite dimensional descriptor system (see, for instance, Reis [26]). This feature implies several difficulties when simulating or tackling the associated optimal control problems, namely in developing efficient methods to solve the Riccati equations appearing in LQR problems. More precisely, after semidiscretization with respect to the space variable, the original problem becomes a LQR problem for a finite dimensional differential algebraic system, which, although projection based efficient numerical methods have been recently developed in the literature, see (for instance, $[8,18,25]$ ), is in general very difficult from the computational viewpoint.

In this paper we study the infinite horizon LQR problem for a system described by the linearized Boussinesq equations with a bounded control operator, by adopting a different methodology, based on the penalization

Keywords and phrases: Linear quadratic optimal control, Riccati theory, Boussinesq system, penalty method, controllability.

Institut de Mathématiques de Bordeaux, 351 Cours de la Libération, 33400 Bordeaux, France.

* Corresponding author: marius.tucsnak@u-bordeaux.fr 
of the free divergence condition. Within this approach, denoting the velocity field of the fluid by $v$ and the pressure field by $p$, the free divergence condition $\operatorname{div} v=0$ is replaced by $\operatorname{div} v+\varepsilon p=0$. As described in the next section, this procedure yields a standard well-posed linear system (governed by a PDE of parabolic type with distributed control). For simulation purposes this approach has been widely used for Stokes, Navier-Stokes or Boussinesq type system. We refer to Temam [31] for the first analysis of the method (for the Navier-Stokes equations) and to Hebeker et al. [17] and Shen [27, 28] for error estimates in the case of non stationary Stokes and Navier-Stokes systems. From an optimal control perspective, the penalty method has been applied in Badra, Buchot and Thevenet [2] for the finite horizon LQR problem where the authors prove convergence results for the penalized Oseen system with boundary control.

To give a more precise view on the questions in the present work, consider the Boussinesq system in a bounded domain $\Omega \subset \mathbb{R}^{d}$, with $d \in\{2,3\}$, whose boundary is denoted by $\Gamma:=\partial \Omega$. It can be written as

$$
\begin{cases}\partial_{t} v-\nu \Delta v+(v \cdot \nabla) v+\nabla p=y e_{d}+f+P \tilde{B} \tilde{u} & \text { in }(0, \infty) \times \Omega, \\ \partial_{t} y-\alpha \Delta y+v \cdot \nabla y=g+B_{d+1} u_{d+1} & \text { in }(0, \infty) \times \Omega, \\ \operatorname{div} v=0 & \text { in }(0, \infty) \times \Omega, \\ v=0, y=0 & \text { on }(0, \infty) \times \Gamma, \\ v(0, \cdot)=v_{0}, y(0, \cdot)=y_{0} & \text { in } \Omega .\end{cases}
$$

In the above equations, $v$ is the velocity field of the fluid, $p$ is the pressure field, $y$ stands for the temperature field and $\left\{e_{1}, e_{2}, \ldots, e_{d}\right\}$ is the standard basis of $\mathbb{R}^{d}$. Moreover, $\nu>0$ is the viscosity parameter, $\alpha>0$ is the heat conductivity in the fluid. The terms $f: \Omega \rightarrow \mathbb{R}^{d}$ and $g: \Omega \rightarrow \mathbb{R}$ describe the influence of field forces and possible internal heat sources. The operator $P:\left[L^{2}(\Omega)\right]^{d} \rightarrow V^{0}(\Omega)$, with (denoting by $n$ the unit normal to $\partial \Omega$ oriented towards the exterior of $\Omega$ )

$$
V^{0}(\Omega)=\left\{v \in L^{2}(\Omega)^{d} ; \operatorname{div} v=0, v \cdot n=0 \text { on } \partial \Omega\right\}
$$

is the Leray projector (see, for instance Sohr [29], Sect. II.2.5). The (bounded) control operator $B:=\left(\tilde{B}, B_{d+1}\right)$ will be required to satisfy various assumptions, which will be described below, whereas $u:=\left(\tilde{u}, u_{d+1}\right)$ is an input function, taking its values in a Hilbert space $U$, called the space of controls.

In this work we consider the linearization of the system (1.1) around a stationary solution $\left(v_{s}, p_{s}, y_{s}\right)$. More precisely, $\left(v_{s}, p_{s}, y_{s}\right)$ is supposed to satisfy the equations

$$
\begin{cases}-\nu \Delta v_{s}+\left(v_{s} \cdot \nabla\right) v_{s}+\nabla p_{s}=y_{s} e_{d}+f & \text { in } \Omega, \\ -\alpha \Delta y_{s}+v_{s} \cdot \nabla y_{s}=g & \text { in } \Omega, \\ \operatorname{div} v_{s}=0 & \text { in } \Omega, \\ v_{s}=0, y_{s}=0 & \text { on } \Gamma,\end{cases}
$$

where the source terms $f$ and $g$ coincide with those in (1.1). It is not difficult to see that the linearization of (1.1) around a stationary state satisfying (1.3) is

$$
\begin{cases}\partial_{t} v-\nu \Delta v+(v \cdot \nabla) v_{s}+\left(v_{s} \cdot \nabla\right) v+\nabla p=y e_{d}+P \tilde{B} \tilde{u} & \text { in }(0, \infty) \times \Omega \\ \partial_{t} y-\alpha \Delta y+v_{s} \cdot \nabla y+v \cdot \nabla y_{s}=B_{d+1} u_{d+1} & \text { in }(0, \infty) \times \Omega, \\ \operatorname{div} v=0 & \text { in }(0, \infty) \times \Omega, \\ v=0, y=0 & \text { on }(0, \infty) \times \Gamma \\ v(0, \cdot)=v_{0}, y(0, \cdot)=y_{0} & \text { in } \Omega .\end{cases}
$$


The goal of this article is to study the approximation of an infinite horizon LQR optimal control problem for (1.4) by the corresponding optimal control problem for the penalized system

$$
\begin{cases}\partial_{t} v_{\varepsilon}-\nu \Delta v_{\varepsilon}+\left(v_{\varepsilon} \cdot \nabla\right) v_{s}+\left(v_{s} \cdot \nabla\right) v_{\varepsilon}+\nabla p_{\varepsilon}=y_{\varepsilon} e_{d}+\tilde{B} \tilde{u} & \text { in }(0, \infty) \times \Omega, \\ \partial_{t} y_{\varepsilon}-\alpha \Delta y_{\varepsilon}+v_{s} \cdot \nabla y_{\varepsilon}+v_{\varepsilon} \cdot \nabla y_{s}=B_{d+1} u_{d+1} & \text { in }(0, \infty) \times \Omega \\ \operatorname{div} v_{\varepsilon}+\varepsilon p_{\varepsilon}=0 & \text { in }(0, \infty) \times \Omega \\ v_{\varepsilon}=0, y_{\varepsilon}=0 & \text { on }(0, \infty) \times \Gamma \\ v_{\varepsilon}(0, \cdot)=v_{0}, y_{\varepsilon}(0, \cdot)=y_{0} & \text { in } \Omega,\end{cases}
$$

where $\varepsilon>0$ is a penalization parameter. Note that the Leray projector $P$ does not appear in (1.5) because the velocity field is not divergence free. By eliminating the pressure in (1.5), we can reformulate the above equations as the following parabolic system:

$$
\begin{cases}\partial_{t} v_{\varepsilon}-\nu \Delta v_{\varepsilon}+\left(v_{\varepsilon} \cdot \nabla\right) v_{s}+\left(v_{s} \cdot \nabla\right) v_{\varepsilon}-\frac{1}{\varepsilon} \nabla \operatorname{div} v_{\varepsilon}=y_{\varepsilon} e_{d}+\tilde{B} \tilde{u} & \text { in }(0, \infty) \times \Omega, \\ \partial_{t} y_{\varepsilon}-\alpha \Delta y_{\varepsilon}+v_{s} \cdot \nabla y_{\varepsilon}+v_{\varepsilon} \cdot \nabla y_{s}=B_{d+1} u_{d+1}, & \text { in }(0, \infty) \times \Omega, \\ v_{\varepsilon}=0, y_{\varepsilon}=0 & \text { on }(0, \infty) \times \Gamma, \\ v_{\varepsilon}(0, \cdot)=v_{0}, y_{\varepsilon}(0, \cdot)=y_{0} & \text { in } \Omega .\end{cases}
$$

For $\left[\begin{array}{l}v_{0} \\ y_{0}\end{array}\right] \in H:=V^{0}(\Omega) \times L^{2}(\Omega)$ and $u \in L^{2}([0, \infty) ; U)$, we introduce the quadratic cost functional

$$
J\left(u ;\left[\begin{array}{l}
v_{0} \\
y_{0}
\end{array}\right]\right)=\int_{0}^{+\infty}\left(\|u(t)\|_{U}^{2}+\left\|\left[\begin{array}{l}
v(t, \cdot) \\
y(t, \cdot)
\end{array}\right]\right\|_{H}^{2}\right) \mathrm{d} t, \text { with } v, p, y \text { subject to (1.4). }
$$

Under suitable assumptions, to be described later, the functional $J$ admits a unique minimum for $u=u_{\mathrm{opt}} \in$ $L^{2}([0, \infty) ; U)$. The corresponding solution of $(1.4)$ is denoted by $\left[\begin{array}{l}v_{\mathrm{opt}} \\ p_{\mathrm{opt}} \\ y_{\mathrm{opt}}\end{array}\right]$ and we have $\left[\begin{array}{l}v_{\mathrm{opt}} \\ y_{\mathrm{opt}}\end{array}\right] \in C([0, \infty) ; H)$. Similarly, for $\left[\begin{array}{l}v_{0} \\ y_{0}\end{array}\right] \in X:=L^{2}(\Omega)^{d+1}$ and $u \in L^{2}([0, \infty) ; U)$, we introduce the cost quadratic functional

$$
J_{\varepsilon}\left(u ;\left[\begin{array}{l}
v_{0} \\
y_{0}
\end{array}\right]\right)=\int_{0}^{+\infty}\left(\|u(t)\|_{U}^{2}+\left\|\left[\begin{array}{l}
v_{\varepsilon}(t, \cdot) \\
y_{\varepsilon}(t, \cdot)
\end{array}\right]\right\|_{X}^{2}\right) \mathrm{d} t, \text { with }\left[\begin{array}{l}
v_{\varepsilon} \\
y_{\varepsilon}
\end{array}\right] \text { subject to (1.6). }
$$

Under suitable assumptions, the functional $J_{\varepsilon}$ admits a unique minimum for $u=u_{\mathrm{opt}, \varepsilon} \in L^{2}([0, \infty) ; U)$, with the corresponding state trajectory $\left[\begin{array}{l}v_{\mathrm{opt}, \varepsilon} \\ y_{\mathrm{opt}, \varepsilon}\end{array}\right] \in C([0, \infty) ; X)$.

The main results of this work assert that, under appropriate assumptions, for every $\left[\begin{array}{l}v_{0} \\ y_{0}\end{array}\right] \in H$ we have

$$
\lim _{\varepsilon \rightarrow 0} u_{\mathrm{opt}, \varepsilon}=u_{\mathrm{opt}} \text { and } \lim _{\varepsilon \rightarrow 0}\left[\begin{array}{l}
v_{\mathrm{opt}, \varepsilon} \\
y_{\mathrm{opt}, \varepsilon}
\end{array}\right]=\left[\begin{array}{l}
v_{\mathrm{opt}} \\
y_{\mathrm{opt}}
\end{array}\right]
$$

in the sense which will be made precise in Theorems 3.1 and 3.2 .

Control problems with an approximation by penalization of the free divergence condition have been studied in different ways. From a controllability point of view, the null-controllability of the Stokes system, respectively Navier-Stokes, is established in [19], respectively in [1], with as many controls as equations. More recently, [6] proves the null-controllability of the Stokes system in 2D with only one control, this result having been extended 
in [7] for the (nonlinear) Boussinesq system in 2D and 3D for cylindrical geometries. For LQR optimal control problems, up to our knowledge, the only article in the literature seems to be [2] where the authors prove results in the spirit of (1.9) for the Oseen system, with boundary control, and for a finite time horizon. We emphasize that the error estimates provided in [2] explode when the horizon time goes to $+\infty$.

The main novelty brought in by this work consists in obtaining convergence results for infinite time horizon LQR problems. An essential ingredient to accomplish this goal is a version of the strategy proposed in Banks and Kunisch [5] for Galerkin approximations, see also Banks and Ito [4]. More precisely, the method essentially consists in the three main steps described below.

1. A question which is common to the various situations we consider is proving that the Riccati operator associated to (1.8) is bounded, uniformly with respect to $\varepsilon$ and that the optimal trajectories decay exponentially, uniformly with respect to $\varepsilon$. This will be shown to hold under two different assumptions.

The first one considers an arbitrary bounded control operator $B$ (possibly of finite rank) and assumes a smallness condition for the stationary state. This is first done for the case of a vanishing stationary state and then by a perturbation argument.

The second situation considered in this work treats the case of a control distributed in a subdomain $\mathcal{O} \subset \Omega$. In this case the control space is infinite dimensional, which enables us to derive uniform null-controllability results for (1.4) and (1.6). This task is accomplished by combining Carleman estimates for the penalized Stokes system due to [1] and Carleman estimates for the heat equation due to [14].

2. Secondly, we prove (1.9) for finite time horizon problems. In order to do this, we first establish convergence results for solutions of (1.6) to solutions of (1.4), in the limit $\varepsilon \rightarrow 0$ and in compact time intervals. This is obtained through an adaptation of the Trotter-Kato theorem and the use of Lax-Phillips semigroups. Then, using the well-known formulas for optimal controls and optimal trajectories, given for instance in [15], we can pass to the limit to deduce (1.9).

3. Finally, by using the uniform bounds and decays that are established in the first point of the strategy, we can approximate the infinite time horizon problem in the interval $[0, \infty)$ by finite time horizon problems in intervals $[-s, 0]$ with $s>0$. By gathering this approximation and the fact that $(1.9)$ holds in $[-s, 0]$, we obtain the expected convergence results (1.9) for the infinite time horizon problem.

The paper is organized as follows. In Section 2, we introduce a functional analytic framework to establish the well-posedness of the control systems (1.4) and (1.6) and we recall some basic facts about LQR problems in infinite horizon. In Section 3 we state the main results of the paper, which basically formalize (1.9) under appropriate assumptions. Section 4 mainly relies on convergence results (in the limit $\varepsilon \rightarrow 0^{+}$) and uniform bounds (in $\varepsilon>0$ ) for semigroups associated to penalized problems. In Section 5 , we prove uniform stabilizability results and null-controllability results for penalized systems. With all these preliminary results at hand, Section 6 is dedicated to the proof of the main results of the paper. Finally, Section 7 contains some final comments and open questions.

\section{SEMigRoup FORMULATION AND BACKGROUND ON LQR PROBLEMS}

For the remaining part of this work we assume that the stationary solution $\left[\begin{array}{l}v_{s} \\ p_{s} \\ y_{s}\end{array}\right]$ of (1.3) verifies

$$
\left[\begin{array}{l}
v_{s} \\
y_{s}
\end{array}\right] \in W^{1, \infty}(\Omega)^{d+1}, \quad p_{s} \in L^{\infty}(\Omega)
$$




\subsection{Well-posedness of the controlled systems}

We introduce below a semigroup formulation of (1.4) and (1.6). To this aim we introduce the spaces

$$
\begin{gathered}
V^{1}(\Omega)=\left\{v \in H_{0}^{1}(\Omega)^{d} ; \operatorname{div} v=0\right\}, \\
\mathcal{D}\left(A_{0}\right)=\left[H^{2}(\Omega)\right]^{d+1} \cap\left[V^{1}(\Omega) \times H_{0}^{1}(\Omega)\right] .
\end{gathered}
$$

Recalling that $H=V^{0}(\Omega) \times L^{2}(\Omega)$ (with $V^{0}(\Omega)$ introduced in (1.2)), we consider the operator $A_{0}: \mathcal{D}\left(A_{0}\right) \rightarrow H$ defined by

$$
A_{0}\left[\begin{array}{l}
\varphi \\
\zeta
\end{array}\right]=\left[\begin{array}{c}
\nu P \Delta \varphi-\left(v_{s} \cdot \nabla\right) \varphi-(\varphi \cdot \nabla) v_{s}+\zeta e_{d} \\
\alpha \Delta \zeta-\left(v_{s} \cdot \nabla \zeta\right)-\left(\varphi \cdot \nabla y_{s}\right)
\end{array}\right] \quad\left((\varphi, \zeta) \in \mathcal{D}\left(A_{0}\right)\right)
$$

where $P$ is the Leray projector. Equivalently, $A_{0}$ can be defined by duality, by setting:

$$
\begin{aligned}
-\left\langle A_{0}\left[\begin{array}{l}
\varphi \\
\zeta
\end{array}\right],\left[\begin{array}{l}
\psi \\
\eta
\end{array}\right]\right\rangle_{H}= & \nu \int_{\Omega} \nabla \varphi: \nabla \bar{\psi} \mathrm{d} x+\int_{\Omega}\left[\left(v_{s} \cdot \nabla\right) \varphi+(\varphi \cdot \nabla) v_{s}\right] \cdot \bar{\psi} \mathrm{d} x-\int_{\Omega} \zeta \overline{\psi_{d}} \mathrm{~d} x \\
& +\alpha \int_{\Omega} \nabla \zeta \cdot \nabla \bar{\eta} \mathrm{d} x+\int_{\Omega}\left[\left(v_{s} \cdot \nabla\right) \zeta+\varphi \cdot \nabla y_{s}\right] \cdot \bar{\eta} \mathrm{d} x \quad\left(\left[\begin{array}{l}
\varphi \\
\zeta
\end{array}\right],\left[\begin{array}{l}
\psi \\
\eta
\end{array}\right] \in \mathcal{D}\left(A_{0}\right)\right) .
\end{aligned}
$$

We will need the result below, for which we sketch the proof, for the sake of completeness, with no claim of originality.

Proposition 2.1. The operator $A_{0}$ defined above generates an analytic semigroup on $H$, denoted by $\mathbb{T}^{0}=$ $\left(\mathbb{T}_{t}^{0}\right)_{t \geqslant 0}$

Proof. By integration by parts and Young's inequalities, it is not difficult to see that there exists a positive constant $c$ depending on $\left\|\left[\begin{array}{l}v_{s} \\ y_{s}\end{array}\right]\right\|_{W^{1, \infty}(\Omega)^{d+1}}$ such that for every $\left[\begin{array}{l}\varphi \\ \psi\end{array}\right] \in \mathcal{D}\left(A_{0}\right)$,

$$
-\left\langle A_{0}\left[\begin{array}{l}
\varphi \\
\zeta
\end{array}\right],\left[\begin{array}{l}
\varphi \\
\zeta
\end{array}\right]\right\rangle_{H} \geqslant \nu\|\nabla \varphi\|_{L^{2}(\Omega)^{d}}^{2}+\alpha\|\nabla \zeta\|_{L^{2}(\Omega)^{d}}^{2}-c\|(\varphi, \zeta)\|_{X}^{2}
$$

where we recall the notation $X=\left[L^{2}(\Omega)\right]^{d+1}$. Thus the corresponding sesquilinear form associated to $-A_{0}+c I$ (this form is simply defined by the right hand side of (2.5)) is coercive on $H$. We also readily check that this form is densely defined, closed and continuous on $H$. By Theorem 1.52 of [21], $A_{0}-c I$ generates an analytic semigroup on $H$ and finally, by a perturbation argument (see, for instance, [22], Sect. 3.2, Cor. 2.2) $A_{0}$ generates an analytic semigroup on $H$.

For every $\varepsilon>0$, consider the operator $A_{\varepsilon}: \mathcal{D}\left(A_{\varepsilon}\right) \rightarrow X$ defined by

$$
\begin{gathered}
\mathcal{D}\left(A_{\varepsilon}\right)=\left[H^{2}(\Omega) \cap H_{0}^{1}(\Omega)\right]^{d+1} \\
A_{\varepsilon}\left[\begin{array}{c}
\varphi \\
\zeta
\end{array}\right]=\left[\begin{array}{c}
\nu \Delta \varphi-\left(v_{s} \cdot \nabla\right) \varphi-(\varphi \cdot \nabla) v_{s}+\frac{1}{\varepsilon} \nabla(\operatorname{div} \varphi)+\zeta e_{d} \\
\alpha \Delta \zeta-\left(v_{s} \cdot \nabla \zeta\right)-\left(\varphi \cdot \nabla y_{s}\right)
\end{array}\right] \quad\left(\left[\begin{array}{l}
\varphi \\
\zeta
\end{array}\right] \in \mathcal{D}\left(A_{\varepsilon}\right)\right) .
\end{gathered}
$$


Alternatively, $A_{\varepsilon}$ can be defined by

$$
\begin{aligned}
-\left\langle A_{\varepsilon}\left[\begin{array}{l}
\varphi \\
\zeta
\end{array}\right],\left[\begin{array}{l}
\psi \\
\eta
\end{array}\right]\right\rangle_{X}= & \nu \int_{\Omega} \nabla \varphi: \nabla \bar{\psi} \mathrm{d} x+\int_{\Omega}\left[\left(v_{s} \cdot \nabla\right) \varphi+(\varphi \cdot \nabla) v_{s}\right] \cdot \bar{\psi} \mathrm{d} x \\
& +\frac{1}{\varepsilon} \int_{\Omega}(\operatorname{div} \varphi)(\operatorname{div} \bar{\psi}) \mathrm{d} x-\int_{\Omega} \zeta \overline{\psi_{d}} \mathrm{~d} x+\alpha \int_{\Omega} \nabla \zeta \cdot \nabla \bar{\eta} \mathrm{d} x \\
& +\int_{\Omega}\left[v_{s} \cdot \nabla \zeta+\varphi \cdot \nabla y_{s}\right] \cdot \bar{\eta} \mathrm{d} x \quad\left(\left[\begin{array}{l}
\varphi \\
\zeta
\end{array}\right],\left[\begin{array}{l}
\psi \\
\eta
\end{array}\right] \in \mathcal{D}\left(A_{\varepsilon}\right)\right) .
\end{aligned}
$$

We have the following result.

Proposition 2.2. For every $\varepsilon>0$ the operator $A_{\varepsilon}$ defined above generates an analytic semigroup on $X=$ $\left[L^{2}(\Omega)\right]^{d+1}$, denoted by $\mathbb{T}^{\varepsilon}=\left(\mathbb{T}_{t}^{\varepsilon}\right)_{t \geqslant 0}$. Moreover, there exist $M \geqslant 1$ and $\omega \in \mathbb{R}$ such that

$$
\left\|\mathbb{T}_{t}^{\varepsilon}\right\|_{\mathcal{L}(X ; X)} \leqslant M \mathrm{e}^{\omega t} \quad(\varepsilon>0, t \geqslant 0) .
$$

Proof. By integration by parts and Young's inequalities, it is not difficult to see that there exists a positive constant $c$ depending on $\left\|\left(v_{s}, y_{s}\right)\right\|_{W^{1, \infty}(\Omega)^{d+1}}$ (independent of $\varepsilon>0$ ), such that for every $\left[\begin{array}{l}\varphi \\ \psi\end{array}\right] \in \mathcal{D}\left(A_{\varepsilon}\right)$,

$$
-\left\langle A_{\varepsilon}\left(\begin{array}{l}
\varphi \\
\zeta
\end{array}\right),\left(\begin{array}{l}
\varphi \\
\zeta
\end{array}\right)\right\rangle_{X} \geqslant \nu\|\nabla \varphi\|_{L^{2}(\Omega)^{d}}^{2}+\frac{1}{\varepsilon}\|\operatorname{div} \varphi\|_{L^{2}(\Omega)}^{2}+\alpha\|\nabla \zeta\|_{L^{2}(\Omega)^{d}}^{2}-c\|(\varphi, \zeta)\|_{X}^{2}
$$

Thus the sesquilinear form associated to $-A_{\varepsilon}+c I$ is coercive on $X$. We also readily check that this form is densely defined, closed and continuous on $X$. By Proposition 5.1 of [21] and Theorem 1.52 of [21], $A_{\varepsilon}-c I$ generates an analytic semigroup $\widetilde{\mathbb{T}^{\varepsilon}}$ satisfying

$$
\left\|\widetilde{\mathbb{T}}_{t}^{\varepsilon}\right\|_{\mathcal{L}(X ; X)} \leqslant M \mathrm{e}^{\omega t} \quad(\varepsilon>0, t \geqslant 0) .
$$

Since the constant $c$ is independent of $\varepsilon$ we can apply ([22], Sect. 3.2, Cor. 2.2), to obtain that for every $\varepsilon>0$ the operator $A_{\varepsilon}$ generates an analytic semigroup on $X$ and that we have (2.9).

Let us introduce the projector $P_{H} \in \mathcal{L}(X ; H)$ defined by

$$
P_{H}\left[\begin{array}{l}
v \\
y
\end{array}\right]=\left[\begin{array}{c}
P v \\
y
\end{array}\right] \quad\left(\left[\begin{array}{l}
v \\
y
\end{array}\right] \in X\right),
$$

where $P$ is the Leray projector. Let $U$ be a Hilbert space (the space of controls). We introduce the control operators

$$
B \in \mathcal{L}(U ; X) \text { and } B_{0}:=P_{H} B \in \mathcal{L}(U ; H) .
$$

We have the following well-posedness result for controlled systems.

Proposition 2.3. For every $\left[\begin{array}{l}v_{0} \\ y_{0}\end{array}\right] \in H, u \in L^{2}([0, \infty) ; U)$, the Cauchy problem

$$
\left[\begin{array}{l}
\dot{v} \\
\dot{y}
\end{array}\right]=A_{0}\left[\begin{array}{l}
v \\
y
\end{array}\right]+B_{0} u, \quad\left[\begin{array}{l}
v(0) \\
y(0)
\end{array}\right]=\left[\begin{array}{l}
v_{0} \\
y_{0}
\end{array}\right],
$$


admits an unique mild solution $\left[\begin{array}{l}v \\ y\end{array}\right]$ defined by

$$
\left[\begin{array}{l}
v(t) \\
y(t)
\end{array}\right]=\mathbb{T}_{t}^{0}\left[\begin{array}{l}
v_{0} \\
y_{0}
\end{array}\right]+\int_{0}^{t} \mathbb{T}_{t-\sigma}^{0} B_{0} u(\sigma) \mathrm{d} \sigma \quad(t \geqslant 0)
$$

Similarly, for every $\left[\begin{array}{l}v_{0} \\ y_{0}\end{array}\right] \in X, u \in L^{2}([0, \infty) ; U)$, the Cauchy problem

$$
\left[\begin{array}{l}
\dot{v} \\
\dot{y}
\end{array}\right]=A_{\varepsilon}\left[\begin{array}{l}
v \\
y
\end{array}\right]+B u, \quad\left[\begin{array}{l}
v(0) \\
y(0)
\end{array}\right]=\left[\begin{array}{l}
v_{0} \\
y_{0}
\end{array}\right]
$$

admits an unique mild solution $(v, y)$, defined by

$$
\left[\begin{array}{l}
v(t) \\
y(t)
\end{array}\right]=\mathbb{T}_{t}^{\varepsilon}\left[\begin{array}{l}
v_{0} \\
y_{0}
\end{array}\right]+\int_{0}^{t} \mathbb{T}_{t-\sigma}^{\varepsilon} B u(\sigma) \mathrm{d} \sigma \quad(t \geqslant 0)
$$

Proof. The proof readily follows from Propositions 2.1 and 2.2 and standard semigroup theoretic results (see, for instance, [32], Prop. 4.2.5).

\subsection{Some background on LQR optimal control problems}

We recall below some basic facts on LQR problems in an infinite dimensional context.

Let $\mathcal{A}$ be the generator of a strongly continuous semigroup on a Hilbert space $\mathcal{X}$ and $\mathcal{B} \in \mathcal{L}(\mathcal{U} ; \mathcal{X})$ be a bounded control operator on a Hilbert space $\mathcal{U}$. The LQR theory in infinite horizon focus on trajectories of the linear control system $x^{\prime}=\mathcal{A} x+\mathcal{B} u, x(0)=x_{0}$, which minimize the quadratic cost functional

$$
\mathcal{J}\left(u ; x_{0}\right)=\int_{0}^{+\infty}\left(\|u(t)\|_{\mathcal{U}}^{2}+\|x(t)\|_{\mathcal{X}}^{2}\right) \mathrm{d} t \quad(u \in \mathcal{U})
$$

We recall below the well-known notion of stabilizability.

Definition 2.4. The pair $(\mathcal{A}, \mathcal{B})$ is stabilizable if there exists $\mathcal{K} \in \mathcal{L}(\mathcal{X}, \mathcal{U})$ such that $\mathcal{A}-\mathcal{B K}$ generates an exponentially stable semigroup $\left(\mathcal{F}_{t}^{\mathcal{K}}\right)_{t \geqslant 0}$ on $\mathcal{X}$, i.e. there exist $M>0$ and $\omega>0$ such that

$$
\left\|\mathcal{F}_{t}^{\mathcal{K}}\right\|_{\mathcal{L}(\mathcal{X} ; \mathcal{X})} \leqslant M e^{-\omega t} \quad(t \geqslant 0) .
$$

One of the main results of the LQR theory states that the optimal control associated to (2.16) is given by a feedback law, see, for instance, Theorem 2.1 of [5] and Theorem 4.2 of [15].

Theorem 2.5. If the pair $(\mathcal{A}, \mathcal{B})$ is stabilizable then for every $x_{0} \in \mathcal{X}, J\left(\cdot ; x_{0}\right)$ admits a unique minimum $u_{\mathrm{opt}}$ given by

$$
u_{\mathrm{opt}}(t)=-\mathcal{B}^{*} \mathcal{P} \mathcal{F}_{t} x_{0} \quad(t \in[0, \infty))
$$

where $P \in \mathcal{L}(\mathcal{X} ; \mathcal{X})$, is the unique nonnegative self-adjoint solution of the Riccati equation

$$
\mathcal{A}^{*} \mathcal{P}+\mathcal{P} \mathcal{A}-\mathcal{P B} \mathcal{B}^{*} \mathcal{P}+I_{\mathcal{X}}=0
$$


and $\mathcal{F}=\left(\mathcal{F}_{t}\right)_{t \geqslant 0}$ is the exponentially stable semigroup on $\mathcal{X}$ generated by $\mathcal{A}-\mathcal{B B}^{*} \mathcal{P}$. Moreover, for every $x_{0} \in \mathcal{X}$,

$$
\min _{u \in L^{2}([0,+\infty) ; \mathcal{U})} \mathcal{J}\left(u ; x_{0}\right)=\left\langle\mathcal{P} x_{0}, x_{0}\right\rangle
$$

We recall that $\mathcal{P} \in \mathcal{L}(\mathcal{X} ; \mathcal{X})$ is a solution to the algebraic Riccati equation $(2.18)$ if $\mathcal{P}$ maps $\mathcal{D}(\mathcal{A})$ to $\mathcal{D}\left(\mathcal{A}^{*}\right)$ and (2.18) is satisfied when the left hand side is applied to an arbitrary $x \in \mathcal{D}(\mathcal{A})$.

\section{MAIN RESUlTS}

In this section we continue to use the notation introduced in Section 2 for the spaces $H, X, U$ and for the operators $\left(A_{\varepsilon}\right)_{\varepsilon \geqslant 0}, B_{0}$ and $B$ (we recall, in particular, from (2.11) that $B_{0}=P_{H} B$ ). Moreover, we introduce some new notation and we state the main results of the paper.

Assume that $\left(A_{0}, B_{0}\right)$, with $A_{0}$ defined in (2.4) and $B_{0}$ defined in (2.11), is stabilizable. By Theorem 2.5, let us denote by $\Pi_{0} \in \mathcal{L}(H)$ the unique nonnegative self-adjoint solution of

$$
A_{0}^{*} \Pi_{0}+\Pi_{0} A_{0}-\Pi_{0} B_{0} B_{0}^{*} \Pi_{0}+I_{H}=0 .
$$

In (3.1), $I_{H}$ denotes the identity operator on $H$. The feedback semigroup, i.e. generated by $A_{0}-B_{0} B_{0}^{*} \Pi_{0}$ is denoted by $\left(\mathbb{F}_{t}^{0}\right)_{t \geqslant 0}$, whereas the optimal control associated to an initial data $\left[\begin{array}{l}v_{0} \\ y_{0}\end{array}\right] \in H$ is given by $u_{\mathrm{opt}, 0}(t)=$ $-B_{0}^{*} \Pi_{0} \mathbb{F}_{t}^{0}\left[\begin{array}{l}v_{0} \\ y_{0}\end{array}\right]$.

In the same way, assume that $\left(A_{\varepsilon}, B\right)$ is stabilizable with $A_{\varepsilon}$ defined in (2.7) and $B$ defined in (2.11). Then let us denote by $\Pi_{\varepsilon} \in \mathcal{L}(X)$ the unique nonnegative self-adjoint solution of

$$
A_{\varepsilon}^{*} \Pi_{\varepsilon}+\Pi_{\varepsilon} A_{\varepsilon}-\Pi_{\varepsilon} B B^{*} \Pi_{\varepsilon}+I_{X}=0 .
$$

In (3.2), $I_{X}$ denotes the identity operator on $X$. The closed loop semigroup, i.e. generated by $A_{\varepsilon}-B B^{*} \Pi_{\varepsilon}$, is denoted by $\left(\mathbb{F}_{t}^{\varepsilon}\right)_{t \geqslant 0}$ and the optimal control, associated to an initial data $\left[\begin{array}{l}v_{0} \\ y_{0}\end{array}\right] \in X$ is given by $u_{\mathrm{opt}, \varepsilon}(t)=$ $-B^{*} \Pi_{\varepsilon} \mathbb{F}_{t}^{\varepsilon}\left[\begin{array}{c}v_{0} \\ y_{0}\end{array}\right]$.

The main results of the article focus on asymptotic properties of $\Pi_{\varepsilon},\left(\mathbb{F}_{t}^{\varepsilon}\right)_{t \geqslant 0}$ and $u_{\mathrm{opt}, \varepsilon}$ in the limit $\varepsilon \rightarrow 0$.

The first main result of the paper treats the case of a stationary state $\left[\begin{array}{l}v_{s} \\ y_{s}\end{array}\right]$ "sufficiently small", with an arbitrary control space $U$ and an arbitrary bounded control operator (possibly of finite rank).

Theorem 3.1. There exists $\delta>0$ such that if

$$
\left\|\left[\begin{array}{l}
v_{s} \\
y_{s}
\end{array}\right]\right\|_{W^{1, \infty}(\Omega)^{d+1}} \leqslant \delta
$$

then $A_{0}$ is exponentially stable and $A_{\varepsilon}$ is uniformly (with respect to $\varepsilon$ ) exponentially stable. Moreover, there exist $M>0$ and $\omega>0$, independent of $\varepsilon$ such that the solution

$$
\begin{gathered}
\left\|\Pi_{\mathcal{E}}\right\|_{\mathcal{L}(X ; X)} \leqslant M \quad(\varepsilon \geqslant 0), \\
\left\|\mathbb{F}_{t}^{\varepsilon}\right\|_{\mathcal{L}(X ; X)} \leqslant M e^{-\omega t} \\
(\varepsilon, t \geqslant 0),
\end{gathered}
$$


In addition, for every $\left[\begin{array}{l}v_{0} \\ y_{0}\end{array}\right] \in H$,

$$
\begin{array}{ll}
\lim _{\varepsilon \rightarrow 0+}\left\|\Pi_{\varepsilon}\left[\begin{array}{l}
v_{0} \\
y_{0}
\end{array}\right]-\Pi_{0}\left[\begin{array}{l}
v_{0} \\
y_{0}
\end{array}\right]\right\|_{H}=0 \quad(t \geqslant 0), \\
\lim _{\varepsilon \rightarrow 0+}\left\|\mathbb{F}_{t}^{\varepsilon}\left[\begin{array}{l}
v_{0} \\
y_{0}
\end{array}\right]-\mathbb{F}_{t}^{0}\left[\begin{array}{l}
v_{0} \\
y_{0}
\end{array}\right]\right\|_{H}=0 & (t \geqslant 0), \\
\lim _{\varepsilon \rightarrow 0+}\left\|u_{\mathrm{opt}, \varepsilon}(t)-u_{\mathrm{opt}, 0}(t)\right\|_{U}=0 & (t \geqslant 0),
\end{array}
$$

and the last two convergences are uniform with respect to $t$ on compact intervals.

Our second main result does no longer require the smallness of $\left(v_{s}, y_{s}\right)$, considering a particular class of control operators.

Theorem 3.2. Assume that $U=X$ and let $B \in \mathcal{L}(U, X)$ be defined by

$$
B u=u \mathbb{1}_{\mathcal{O}} \quad(u \in U),
$$

where $\mathcal{O}$ is an open subset of $\Omega$ and $\mathbb{1}_{\mathcal{O}}$ is the characteristic function of $\mathcal{O}$. Then $\left(A_{0}, B\right)$ is exponentially stabilizable and there exists $\varepsilon_{0}>0$ such that $\left(A_{\varepsilon}, B\right)$ is uniformly, with respect to $\varepsilon \in\left(0, \varepsilon_{0}\right)$, exponentially stabilizable. The uniform bounds (3.4) and (3.5) still hold true for $\varepsilon \in\left(0, \varepsilon_{0}\right)$. Moreover, the corresponding Riccati operators, closed loop generators and optimal LQR controls still satisfy (3.6), (3.7) and (3.8).

\section{Preliminary Results on the (Penalized) Boussinesq Semigroup}

In this section and the following ones we continue to use the notation $H:=V^{0}(\Omega) \times L^{2}(\Omega)$ and $X=$ $\left[L^{2}(\Omega)\right]^{d+1}$ for the state space of the system described by the Boussinesq equations and their penalized version, respectively. We recall that the operator $A_{0}: \mathcal{D}\left(A_{0}\right) \rightarrow H$ has been defined in (2.4), whereas for every $\varepsilon>0$ the operator $A_{\varepsilon}$ has been defined in (2.7). As in the previous sections, the analytic semigroup on $H$ generated by $A_{0}$ is denoted by $\mathbb{T}^{0}$, whereas, for every $\varepsilon>0, \mathbb{T}^{\varepsilon}$ stands for the analytic semigroup on $X$ generated by $A_{\varepsilon}$.

\subsection{Convergence results for the semigroups describing the free dynamics}

In this subsection we prove that when $\varepsilon \rightarrow 0+$ the family of semigroups $\left(\mathbb{T}^{\varepsilon}\right)_{\varepsilon \geqslant 0}$ strongly converges to $\mathbb{T}^{0}$ (and the same for adjoint semigroups). The proof relies on an adaptation of the Trotter-Kato's theorem, described below.

We begin by noticing that the adjoint $A_{0}^{*}$ of $A_{0}$ is defined by $\mathcal{D}\left(A_{0}^{*}\right)=\mathcal{D}\left(A_{0}\right)$ and

$$
A_{0}^{*}\left[\begin{array}{l}
\varphi \\
\zeta
\end{array}\right]=\left[\begin{array}{c}
\nu \Delta \varphi+(\nabla \varphi)^{\operatorname{tr}} v_{s}+(\nabla \varphi) v_{s}-\zeta \nabla y_{s} \\
\alpha \Delta \zeta+\left(v_{s} \cdot \nabla \zeta\right)+\varphi_{d}
\end{array}\right] \quad\left(\left[\begin{array}{c}
\varphi \\
\zeta
\end{array}\right] \in \mathcal{D}\left(A_{0}^{*}\right)\right)
$$

In the same way, for every $\varepsilon>0$ the adjoint $A_{\varepsilon}^{*}$ of $A_{\varepsilon}$ is defined by $\mathcal{D}\left(A_{\varepsilon}^{*}\right)=\mathcal{D}\left(A_{\varepsilon}\right)$ and

$$
A_{\varepsilon}^{*}\left[\begin{array}{c}
\varphi \\
\zeta
\end{array}\right]=\left[\begin{array}{c}
\nu \Delta \varphi+(\nabla \varphi)^{\operatorname{tr}} v_{s}+(\nabla \varphi) v_{s}-\zeta \nabla y_{s}+\frac{1}{\varepsilon} \nabla(\operatorname{div} \varphi) \\
\alpha \Delta \zeta+\left(v_{s} \cdot \nabla \zeta\right)+\varphi_{d}
\end{array}\right] \quad\left(\left[\begin{array}{l}
\varphi \\
\zeta
\end{array}\right] \in \mathcal{D}\left(A_{\varepsilon}^{*}\right)\right)
$$

In $(4.1),(4.2)$, the notation $(\nabla \varphi)^{\operatorname{tr}}$ means the transpose of the matrix $\nabla \varphi$. 
We first prove the convergence of the resolvents of $A_{\varepsilon}$ (respectively $A_{\varepsilon}^{*}$ ) towards the resolvents of $A_{0}$ (respectively $A_{0}^{*}$ ).

Proposition 4.1. There exists $\lambda_{0}>0$ such that for every $\lambda \in \mathbb{C}$ with $\operatorname{Re} \lambda \geqslant \lambda_{0}$, we have

$$
\begin{aligned}
& \lim _{\varepsilon \rightarrow 0}\left\|\left[\lambda I-A_{\varepsilon}\right]^{-1}\left[\begin{array}{l}
f \\
g
\end{array}\right]-\left[\lambda I-A_{0}\right]^{-1} P_{H}\left[\begin{array}{l}
f \\
g
\end{array}\right]\right\|_{X}=0 \quad\left(\left[\begin{array}{l}
f \\
g
\end{array}\right] \in X\right), \\
& \lim _{\varepsilon \rightarrow 0}\left\|\left[\lambda I-A_{\varepsilon}^{*}\right]^{-1}\left[\begin{array}{l}
f \\
g
\end{array}\right]-\left[\lambda I-A_{0}^{*}\right]^{-1} P_{H}\left[\begin{array}{l}
f \\
g
\end{array}\right]\right\|_{X}=0 \quad\left(\left[\begin{array}{l}
f \\
g
\end{array}\right] \in X\right) .
\end{aligned}
$$

Proof. We only prove (4.3), since the proof of (4.4) is fully similar.

Let $\left[\begin{array}{l}f \\ g\end{array}\right] \in X$ and

$$
\lambda_{0}>c>0
$$

where the constant $c$ is the one appearing in (2.10). Then, for $\lambda \in \mathbb{C}$ with $\operatorname{Re} \lambda \geqslant \lambda_{0}$, by setting

$$
\left[\begin{array}{l}
\varphi_{\varepsilon} \\
\zeta_{\varepsilon}
\end{array}\right]=\left(\lambda I-A_{\varepsilon}\right)^{-1}\left[\begin{array}{l}
f \\
g
\end{array}\right]
$$

and by using (2.8) we have

$$
\begin{aligned}
& \lambda \int_{\Omega} \varphi_{\varepsilon} \cdot \bar{\psi} \mathrm{d} x+\nu \int_{\Omega} \nabla \varphi_{\varepsilon}: \nabla \bar{\psi} \mathrm{d} x+\int_{\Omega}\left[\left(v_{s} \cdot \nabla\right) \varphi_{\varepsilon}+\left(\varphi_{\varepsilon} \cdot \nabla\right) v_{s}\right] \cdot \bar{\psi} \mathrm{d} x \\
& +\frac{1}{\varepsilon} \int_{\Omega}\left(\operatorname{div} \varphi_{\varepsilon}\right)(\operatorname{div} \bar{\psi}) \mathrm{d} x-\int_{\Omega} \zeta_{\varepsilon} \overline{\psi_{d}} \mathrm{~d} x+\lambda \int_{\Omega} \zeta_{\varepsilon} \bar{\eta} \mathrm{d} x+\alpha \int_{\Omega} \nabla \zeta_{\varepsilon} \cdot \nabla \bar{\eta} \mathrm{d} x \\
& +\int_{\Omega}\left[v_{s} \cdot \nabla \zeta_{\varepsilon}+\varphi_{\varepsilon} \cdot \nabla y_{s}\right] \cdot \bar{\eta} \mathrm{d} x=\int_{\Omega} f \cdot \bar{\psi} \mathrm{d} x+\int_{\Omega} g \bar{\eta} \mathrm{d} x \quad\left(\left[\begin{array}{l}
\psi \\
\eta
\end{array}\right] \in \mathcal{D}\left(A_{\varepsilon}\right)\right) .
\end{aligned}
$$

Taking $\left[\begin{array}{l}\psi \\ \eta\end{array}\right]=\left[\begin{array}{l}\varphi_{\varepsilon} \\ \zeta_{\varepsilon}\end{array}\right]$ in (4.6) and using (2.10) we obtain

$$
\left\|\nabla \varphi_{\varepsilon}\right\|_{L^{2}(\Omega)^{d}}^{2}+\frac{1}{\varepsilon}\left\|\operatorname{div} \varphi_{\varepsilon}\right\|_{L^{2}(\Omega)}^{2}+\left\|\nabla \zeta_{\varepsilon}\right\|_{L^{2}(\Omega)^{d}}^{2} \leqslant c\left\|\left[\begin{array}{l}
f \\
g
\end{array}\right]\right\|_{X}^{2} \quad(\varepsilon>0),
$$

where $c>0$ is some another constant. From this and the Poincaré inequality it follows that there exists (another) $c>0$ with

$$
\left\|\varphi_{\varepsilon}\right\|_{H_{0}^{1}(\Omega)^{d}}^{2}+\frac{1}{\varepsilon}\left\|\operatorname{div} \varphi_{\varepsilon}\right\|_{L^{2}(\Omega)}^{2}+\left\|\zeta_{\varepsilon}\right\|_{H_{0}^{1}(\Omega)}^{2} \leqslant c\left\|\left[\begin{array}{l}
f \\
g
\end{array}\right]\right\|_{X}^{2} \quad(\varepsilon>0) .
$$

The above estimate implies that there exists $\left[\begin{array}{l}\varphi \\ \zeta\end{array}\right] \in H_{0}^{1}(\Omega)^{d+1}$ such that

$$
\left[\begin{array}{c}
\varphi_{\varepsilon} \\
\zeta_{\varepsilon}
\end{array}\right] \rightarrow\left[\begin{array}{l}
\varphi \\
\zeta
\end{array}\right] \text { as } \varepsilon \rightarrow 0 \text { in } H_{0}^{1}(\Omega)^{d+1} \text { weakly }
$$




$$
\left[\begin{array}{c}
\varphi_{\varepsilon} \\
\zeta_{\varepsilon}
\end{array}\right] \rightarrow\left[\begin{array}{l}
\varphi \\
\zeta
\end{array}\right] \text { as } \varepsilon \rightarrow 0 \text { in } L^{2}(\Omega)^{d+1} \text { strongly }
$$

$$
\operatorname{div} \varphi=0
$$

For $\left[\begin{array}{l}\psi \\ \eta\end{array}\right] \in \mathcal{D}\left(A_{0}\right)$, note that $\operatorname{div} \psi=0$, we can thus pass to the limit in (4.6) to obtain that

$$
\begin{aligned}
& \lambda \int_{\Omega} \varphi \cdot \bar{\psi} \mathrm{d} x+\nu \int_{\Omega} \nabla \varphi: \nabla \bar{\psi} \mathrm{d} x+\int_{\Omega}\left[\left(v_{s} \cdot \nabla\right) \varphi+(\varphi \cdot \nabla) v_{s}\right] \cdot \bar{\psi} \mathrm{d} x \\
& -\int_{\Omega} \zeta \overline{\psi_{d}} \mathrm{~d} x+\lambda \int_{\Omega} \zeta \bar{\eta} \mathrm{d} x+\alpha \int_{\Omega} \nabla \zeta \cdot \nabla \bar{\eta} \mathrm{d} x \\
& +\int_{\Omega}\left[v_{s} \cdot \nabla \zeta+\varphi \cdot \nabla y_{s}\right] \cdot \bar{\eta} \mathrm{d} x=\int_{\Omega} f \cdot \bar{\psi} \mathrm{d} x+\int_{\Omega} g \bar{\eta} \mathrm{d} x, \quad\left(\left[\begin{array}{l}
\psi \\
\eta
\end{array}\right] \in \mathcal{D}\left(A_{0}\right)\right),
\end{aligned}
$$

then using

$$
\int_{\Omega} f \cdot \bar{\psi} \mathrm{d} x+\int_{\Omega} g \bar{\eta} \mathrm{d} x=\int_{\Omega} P_{H}\left[\begin{array}{l}
f \\
g
\end{array}\right] \cdot\left[\begin{array}{l}
\psi \\
\eta
\end{array}\right] \mathrm{d} x \quad\left(\left[\begin{array}{l}
\psi \\
\eta
\end{array}\right] \in \mathcal{D}\left(A_{0}\right)\right)
$$

we deduce (4.3) from (4.7).

Note that, arguing as in Proposition 2.1 and in Proposition 2.2, the operator $A_{0}^{*}$ generates an analytic semigroup on $H$, denoted by $\left(\mathbb{T}^{0}\right)^{*}=\left(\left(\mathbb{T}_{t}^{0}\right)^{*}\right)_{t \geqslant 0}$ and for every $\varepsilon>0$ the operator $A_{\varepsilon}^{*}$ generates an analytic semigroup on $X$, denoted by $\left(\mathbb{T}^{\varepsilon}\right)^{*}=\left(\left(\mathbb{T}_{t}^{\varepsilon}\right)^{*}\right)_{t \geqslant 0}$. Moreover, there exist $M \geqslant 1$ and $\omega \in \mathbb{R}$ such that

$$
\left\|\left(\mathbb{T}_{t}^{\varepsilon}\right)^{*}\right\|_{\mathcal{L}(X ; X)} \leqslant M \mathrm{e}^{\omega t} \quad(\varepsilon>0, t \geqslant 0)
$$

An important consequence of Proposition 4.1 is the following result:

Proposition 4.2. For every $\left[\begin{array}{l}v_{0} \\ y_{0}\end{array}\right] \in H$, we have

$$
\begin{gathered}
\lim _{\varepsilon \rightarrow 0}\left\|\mathbb{T}_{t}^{\varepsilon}\left[\begin{array}{l}
v_{0} \\
y_{0}
\end{array}\right]-\mathbb{T}_{t}^{0}\left[\begin{array}{l}
v_{0} \\
y_{0}
\end{array}\right]\right\|_{X}=0 \quad(t \geqslant 0), \\
\lim _{\varepsilon \rightarrow 0}\left\|\left(\mathbb{T}_{t}^{\varepsilon}\right)^{*}\left[\begin{array}{l}
v_{0} \\
y_{0}
\end{array}\right]-\left(\mathbb{T}_{t}^{0}\right)^{*}\left[\begin{array}{l}
v_{0} \\
y_{0}
\end{array}\right]\right\|_{X}=0 \quad(t \geqslant 0),
\end{gathered}
$$

uniformly with respect to $t$ on compact intervals.

Proof. We only prove (4.10). The proof of (4.11) follows the same scheme, using (4.4) instead of (4.3).

We follow Section 3.4, Theorem 4.2 of [22]. We take $\lambda \geqslant \lambda_{0}$ where $\lambda_{0}$ is defined in Proposition 4.1 and we note

$$
R_{\lambda}^{\varepsilon}=\left[\lambda I-A_{\varepsilon}\right]^{-1}, R_{\lambda}^{0}=\left[\lambda I-A_{0}\right]^{-1}
$$


By using that the resolvent commutes with the associated semigroup, see Section 1.2, Theorem 2.4, c) of [22], we have

$$
\begin{aligned}
\left\|\left(\mathbb{T}_{t}^{\varepsilon}-\mathbb{T}_{t}^{0}\right) R_{\lambda}^{0} z_{0}\right\|_{X} & \leqslant\left\|\mathbb{T}_{t}^{\varepsilon}\left(R_{\lambda}^{0}-R_{\lambda}^{\varepsilon}\right) z_{0}\right\|_{X}+\left\|R_{\lambda}^{\varepsilon}\left(\mathbb{T}_{t}^{\varepsilon}-\mathbb{T}_{t}^{0}\right) z_{0}\right\|_{X}+\left\|\left(R_{\lambda}^{\varepsilon}-R_{\lambda}^{0}\right) \mathbb{T}_{t}^{0} z_{0}\right\|_{X} \\
& =: D_{1}^{\varepsilon} z_{0}+D_{2}^{\varepsilon} z_{0}+D_{3}^{\varepsilon} z_{0} \quad\left(\varepsilon>0, t \in[0, T], z_{0} \in H\right) .
\end{aligned}
$$

It follows from (4.3) and (2.9) that $D_{1}^{\varepsilon} z_{0} \rightarrow 0$ as $\varepsilon \rightarrow 0$, uniformly on $[0, T]$. Also, since $t \in[0, T] \mapsto \mathbb{T}_{t}^{0} z_{0}$ is continuous because $\mathbb{T}^{0}$ is a strongly continuous semigroup, we deduce that the set $\left\{\mathbb{T}_{t}^{0} z_{0} ; t \in[0, T]\right\}$ is compact in $H$ and therefore $D_{3}^{\varepsilon} z_{0} \rightarrow 0$ as $\varepsilon \rightarrow 0$ uniformly on $[0, T]$.

For the term $D_{2}^{\varepsilon} z_{0}$, we use the fact, proved in Section 3.4, Lemma 4.1 of [22], that

$$
R_{\lambda}^{\varepsilon}\left(\mathbb{T}_{t}^{\varepsilon}-\mathbb{T}_{t}^{0}\right) R_{\lambda}^{0} z_{0}=-\int_{0}^{t} \mathbb{T}_{t-s}^{\varepsilon}\left(R_{\lambda}^{0}-R_{\lambda}^{\varepsilon}\right) \mathbb{T}_{s}^{0} z_{0} \mathrm{~d} s \quad\left(\varepsilon>0, t \in[0, T], z_{0} \in H\right) .
$$

From (4.12), we have

$$
\begin{aligned}
\left\|R_{\lambda}^{\varepsilon}\left(\mathbb{T}_{t}^{\varepsilon}-\mathbb{T}_{t}^{0}\right) R_{\lambda}^{0} z_{0}\right\|_{X} & \leqslant \int_{0}^{t}\left\|\mathbb{T}_{t-s}^{\varepsilon}\left(R_{\lambda}^{0}-R_{\lambda}^{\varepsilon}\right) \mathbb{T}_{s}^{0} z_{0}\right\|_{X} \mathrm{~d} s \\
& \leqslant \int_{0}^{t} M e^{\omega T}\left\|\left(R_{\lambda}^{0}-R_{\lambda}^{\varepsilon}\right) \mathbb{T}_{s}^{0} z_{0}\right\|_{X} \mathrm{~d} s \quad\left(\varepsilon>0, t \in[0, T], z_{0} \in H\right) .
\end{aligned}
$$

The integrand in the right hand side of the above estimate is bounded by an independent function of $s$ and tends to 0 as $\varepsilon \rightarrow 0$ by using (4.3). So, by Lebesgue's convergence theorem, we have

$$
\left\|R_{\lambda}^{\varepsilon}\left(\mathbb{T}_{t}^{\varepsilon}-\mathbb{T}_{t}^{0}\right) R_{\lambda}^{0} z_{0}\right\|_{X} \rightarrow 0 \text { as } \varepsilon \rightarrow 0 \quad\left(t \in[0, T], z_{0} \in H\right),
$$

and the limit is uniform in $[0, T]$.

So we have that $D_{2}^{\varepsilon} R_{\lambda}^{0} z_{0} \rightarrow 0$ as $\varepsilon \rightarrow 0$ uniformly in $[0, T]$ for every $z_{0} \in H$. Moreover, we know that every $z_{0} \in \mathcal{D}\left(A_{0}\right)$ can be written as $z_{0}=R_{\lambda}^{0} z_{1}$ with $z_{1} \in H$ so we deduce that $D_{2}^{\varepsilon} z_{0} \rightarrow 0$ as $\varepsilon \rightarrow 0$ uniformly in $[0, T]$ for every $z_{0} \in \mathcal{D}\left(A_{0}\right)$. Then, we have actually proved that

$$
\left\|\left(\mathbb{T}_{t}^{\varepsilon}-\mathbb{T}_{t}^{0}\right) R_{\lambda}^{0} z_{0}\right\|_{X} \rightarrow 0 \text { as } \varepsilon \rightarrow 0 \quad\left(t \in[0, T], z_{0} \in \mathcal{D}\left(A_{0}\right)\right),
$$

so we deduce that

$$
\left\|\left(\mathbb{T}_{t}^{\varepsilon}-\mathbb{T}_{t}^{0}\right) z_{0}\right\|_{X} \rightarrow 0 \text { as } \varepsilon \rightarrow 0 \quad\left(t \in[0, T], z_{0} \in \mathcal{D}\left(A_{0}^{2}\right)\right)
$$

Moreover the above convergence is uniform on $[0, T]$. By using that $\left\|\mathbb{T}_{t}^{\varepsilon}-\mathbb{T}_{t}^{0}\right\|$ is uniformly bounded on $[0, T]$ and since $\mathcal{D}\left(A_{0}^{2}\right)$ is dense in $H$, see Theorem 1.2.7 of [22], we have that (4.13) holds for every $z_{0} \in H$ and uniformly for $t \in[0, T]$, which concludes the proof.

\subsection{Lax-Phillips semigroups}

In this section we recall, following Staffans and Weiss [30], the concept of Lax-Phillips semigroups associated to a control system which is then used, combined with the Trotter-Kato theorem, to prove the convergence of the penalized Boussinesq control systems towards the usual one.

Let $\mathcal{A}: \mathcal{D}(\mathcal{A}) \rightarrow \mathcal{X}$ be the generator of a strongly continuous semigroup $\mathcal{F}=\left(\mathcal{F}_{t}\right)_{t \geqslant 0}$. We denote by $\omega_{\mathcal{F}}$ the growth bound of $\mathcal{F}$ and we fix $\omega>\omega_{\mathcal{F}}$. Let $\mathcal{B} \in \mathcal{L}(\mathcal{U}, \mathcal{X})$ be a bounded control operator. We denote

$$
\mathcal{U}_{\omega}=L_{\omega}^{2}([0, \infty) ; \mathcal{U}):=\mathrm{e}_{\omega} L^{2}([0, \infty) ; \mathcal{U}),
$$


where $\left(\mathrm{e}_{\omega} v\right)(t)=e^{\omega t} v(t)$ and $\left\|\mathrm{e}_{\omega} v\right\|_{L_{\omega}^{2}}=\|v\|_{L^{2}}$. For later use, we also introduce the notation

$$
W_{\omega}^{m, 2}([0, \infty) ; \mathcal{U}):=\mathrm{e}_{\omega} W^{m, 2}([0, \infty) ; \mathcal{U})
$$

with $\left\|\mathrm{e}_{\omega} v\right\|_{W_{\omega}^{m, 2}}=\|v\|_{W^{m, 2}}$.

Let

$$
V=\mathcal{D}(\mathcal{A}) \times \mathcal{U}_{\omega}
$$

It is known (see Grabowski and Callier [16] for the first derivation of this result and Staffans and Weiss [30] for a presentation of these results in a much more general context), that the operator $\mathcal{C}: \mathcal{D}(\mathcal{A}) \rightarrow \mathcal{X} \times \mathcal{U}_{\omega}$ defined by

$$
\begin{gathered}
\mathcal{D}(\mathcal{C})=\mathcal{D}(A) \times W_{\omega}^{1,2}((0, \infty) ; \mathcal{U}), \\
\mathcal{C}=\left[\begin{array}{cc}
\mathcal{A} & \mathcal{B} \delta_{0} \\
0 & \frac{\mathrm{d}}{\mathrm{d} \xi}
\end{array}\right]
\end{gathered}
$$

generates a strongly continuous semigroup $\Xi$ on $\mathcal{X} \times \mathcal{U}_{\omega}$. More precisely, we have

$$
\boldsymbol{\Xi}_{t}=\left[\begin{array}{cc}
\mathcal{F}_{t} & \Phi_{t} \\
0 & S_{t}^{*}
\end{array}\right] \quad(t \geqslant 0)
$$

where $\left(\Phi_{t}\right)_{t \geqslant 0}$ are the output maps defined by

$$
\Phi_{t} u=\int_{0}^{t} \mathcal{F}_{t-\sigma} \mathcal{B} u(\sigma) \mathrm{d} \sigma \quad\left(t \geqslant 0, u \in \mathcal{U}_{\omega}\right)
$$

and $S_{t}^{*}$ is the left shift by $t$ on $L_{\text {loc }}^{2}([0, \infty) ; \mathcal{U})$, i.e.,

$$
S_{t}^{*} u(\tau)=u(\tau+t) \quad\left(t, \tau>0, u \in L_{\mathrm{loc}}^{2}([0, \infty) ; \mathcal{U})\right) .
$$

Remark 4.3. Note that, as mentioned in [30], the growth bound of $\Xi$ equals to $\omega$.

Proposition 4.4. Let $\mathcal{C}$ be the operator defined in (4.15) and (4.16). Then its resolvent set $\rho(\mathcal{C})$ contains the half plane

$$
\mathbb{C}_{\omega}=\{\lambda \in \mathbb{C} \mid \operatorname{Re} \lambda>\omega\},
$$

and we have

$$
(\lambda I-\mathcal{C})^{-1}\left[\begin{array}{c}
z \\
u
\end{array}\right]=\left[\begin{array}{c}
(\lambda I-\mathcal{A})^{-1}\left(\mathcal{B} \int_{0}^{\infty} e^{-\lambda \eta} u(\eta) \mathrm{d} \eta+z\right) \\
\xi \mapsto-\int_{\xi}^{\infty} e^{\lambda(\xi-\eta)} u(\eta) \mathrm{d} \eta
\end{array}\right] \quad\left(\lambda \in \mathbb{C}_{\omega}, z \in \mathcal{X}, u \in \mathcal{U}_{\omega}\right)
$$

Proof. Given $\lambda \in \mathbb{C}_{\omega}$, the equation

$$
(\lambda I-\mathcal{C})\left[\begin{array}{l}
z_{0} \\
u_{0}
\end{array}\right]=\left[\begin{array}{l}
z \\
u
\end{array}\right]
$$


writes

$$
\begin{gathered}
(\lambda I-\mathcal{A}) z_{0}+\mathcal{B} u_{0}(0)=z, \\
\lambda u_{0}-\frac{\mathrm{d} u_{0}}{\mathrm{~d} \xi}=u .
\end{gathered}
$$

Denoting $u_{-\omega}=\mathrm{e}_{-\omega} u$ and $u_{0,-\omega}=\mathrm{e}_{-\omega} u_{0}$, we note that $u_{-\omega} \in L^{2}([0, \infty) ; \mathcal{U})$ and that $u_{0} \in W_{\omega}^{1,2}([0, \infty) ; \mathcal{U})$ if and only if $u_{0,-\omega} \in W^{1,2}([0, \infty) ; \mathcal{U})$. Moreover, equation (4.20) can be rephrased in terms of $u_{-\omega}$ and $u_{0,-\omega}$ as

$$
\frac{\mathrm{d} u_{0,-\omega}}{\mathrm{d} \xi}(\xi)=(\lambda-\omega) u_{0,-\omega}(\xi)+u_{-\omega}(\xi) \quad(\operatorname{Re} \lambda>\omega, \xi \in(0, \infty))
$$

In the following, we use some basic facts about Laplace transformation, see for instance Section 12.4 of [32]. By applying the Laplace transform to all the terms in the above equation we obtain

$$
s \widehat{u}_{0,-\omega}(s)-u_{0,-\omega}(0)=(\lambda-\omega) \widehat{u}_{0,-\omega}(s)+\widehat{u}_{-\omega}(s) \quad(\operatorname{Re} s>0, \operatorname{Re} \lambda>\omega) .
$$

Choosing $u_{0,-\omega}(0)=-\widehat{u}_{-\omega}(\lambda-\omega)$ we obtain

$$
\widehat{u}_{0,-\omega}(s)=\frac{\widehat{u}_{-\omega}(s)-\widehat{u}_{-\omega}(\lambda-\omega)}{s-(\lambda-\omega)} \quad(\operatorname{Re} s>0, \operatorname{Re} \lambda>\omega) .
$$

Since, by the Paley-Wiener theorem, see Theorem 12.4.2 of [32], we have that $\widehat{u}_{-\omega}$ lies in the Hardy space $\mathcal{H}^{2}\left(\mathbb{C}_{0} ; \mathcal{U}\right)$, the above formula implies that $\widehat{u}_{0,-\omega} \in \mathcal{H}^{2}\left(\mathbb{C}_{0} ; \mathcal{U}\right)$. Applying again the Paley-Wiener theorem it follows that $\widehat{u}_{0,-\omega}$ is the unique solution of $(4.21)$ with $u_{0,-\omega} \in L^{2}([0, \infty) ; \mathcal{U})$. Moreover, applying the inverse Laplace transform to (4.22), we obtain that

$$
u_{0,-\omega}(\xi)=-e^{(\lambda-\omega) \xi} \int_{\xi}^{\infty} \mathrm{e}^{(\omega-\lambda) \eta} \tilde{u}(\eta) \mathrm{d} \eta \quad(\operatorname{Re} \lambda>\omega, \xi \in(0, \infty))
$$

so that

$$
u_{0}(\xi)=-\int_{\xi}^{\infty} e^{\lambda(\xi-\eta)} u(\eta) \mathrm{d} \eta \quad(\operatorname{Re} \lambda>\omega, \xi \in(0, \infty))
$$

Going back to (4.19), it follows that

$$
z_{0}=(\lambda I-\mathcal{A})^{-1}\left(-u_{0}(0)+z\right) \quad(\operatorname{Re} \lambda>\omega) .
$$

Finally, putting together (4.23) and (4.24) we obtain the conclusion (4.17).

Let us now retrieve our (penalized) Boussinesq context, with the notation for the operators $A_{\varepsilon}$ and semigroups $\mathbb{T}^{\varepsilon}$ recalled at the beginning of this section. We know from Proposition 2.1 and Proposition 2.2 that there exist $M \geqslant 1$ and $\tilde{\omega} \in \mathbb{R}$ such that the corresponding semigroups satisfy

$$
\left\|\mathbb{T}_{t}^{0}\right\|_{\mathcal{L}(X ; X)} \leqslant M e^{\tilde{\omega} t} \text { and } \quad\left\|\mathbb{T}_{t}^{\varepsilon}\right\|_{\mathcal{L}(X ; X)} \leqslant M e^{\tilde{\omega} t}, \quad(\varepsilon>0, t \geqslant 0) .
$$

For $\omega>\tilde{\omega}$, we consider the space $\mathcal{U}_{\omega}$ introduced in (4.14) taking $\mathcal{U}=U$. 
We can introduce the Lax-Phillips semigroup $\Xi^{0}=\left(\Xi_{t}^{0}\right)_{t \geqslant 0}$ on $H \times \mathcal{U}_{\omega}$, associated to the pair $\left(A_{0}, B_{0}=P_{H} B\right)$ and, for every $\varepsilon>0$, the Lax-Phillips semigroup $\Xi^{\varepsilon}=\left(\Xi_{t}^{\varepsilon}\right)_{t \geqslant 0}$ on $X \times \mathcal{U}_{\omega}$, associated to the pair $\left(A_{\varepsilon}, B\right)$.

The main result in this section is given just below and it will be used several times in the remaining part of this work.

Proposition 4.5. With the above notation, we have

$$
\begin{aligned}
& \lim _{\varepsilon \rightarrow 0+}\left\|\Xi_{t}^{\varepsilon}\left[\begin{array}{l}
z \\
u
\end{array}\right]-\Xi_{t}^{0}\left[\begin{array}{c}
z \\
u
\end{array}\right]\right\|_{X \times \mathcal{U}}=0\left(t \geqslant 0,\left[\begin{array}{c}
z \\
u
\end{array}\right] \in H \times \mathcal{U}_{\omega}\right), \\
& \lim _{\varepsilon \rightarrow 0+}\left\|\left(\Xi_{t}^{\varepsilon}\right)^{*}\left[\begin{array}{c}
z \\
u
\end{array}\right]-\left(\Xi_{t}^{0}\right)^{*}\left[\begin{array}{l}
z \\
u
\end{array}\right]\right\|_{X \times \mathcal{U}}=0 \quad\left(t \geqslant 0,\left[\begin{array}{c}
z \\
u
\end{array}\right] \in H \times \mathcal{U}_{\omega}\right),
\end{aligned}
$$

Moreover, the convergence above is uniform for $t$ in compact intervals.

Proof. We only prove (4.26).

The generator of $\Xi^{0}$ is the operator $\mathcal{C}_{0}: \mathcal{D}\left(\mathcal{C}_{0}\right) \rightarrow H \times \mathcal{U}_{\omega}$ defined by

$$
\begin{gathered}
\mathcal{D}\left(\mathcal{C}_{0}\right)=\mathcal{D}\left(A_{0}\right) \times W_{\omega}^{1,2}((0, \infty) ; U), \\
\mathcal{C}_{0}=\left[\begin{array}{cc}
A_{0} & B_{0} \delta_{0} \\
0 & \frac{\mathrm{d}}{\mathrm{d} \xi}
\end{array}\right] .
\end{gathered}
$$

Similarly, for every $\varepsilon>0$, the generator of $\Xi^{\varepsilon}$ is the operator $\mathcal{C}_{\varepsilon}: \mathcal{D}\left(\mathcal{C}_{\varepsilon}\right) \rightarrow X \times \mathcal{U}$ defined by

$$
\begin{gathered}
\mathcal{D}\left(\mathcal{C}_{\varepsilon}\right)=\mathcal{D}\left(A_{\varepsilon}\right) \times W_{\omega}^{1,2}((0, \infty) ; U), \\
\mathcal{C}_{\varepsilon}=\left[\begin{array}{cc}
A_{\varepsilon} & B \delta_{0} \\
0 & \frac{\mathrm{d}}{\mathrm{d} \xi}
\end{array}\right] .
\end{gathered}
$$

By Proposition 4.4, we have for every $\lambda \in \mathbb{C}_{\omega}$,

$$
\begin{aligned}
& \left(\lambda I-\mathcal{C}_{0}\right)^{-1}\left[\begin{array}{l}
z \\
u
\end{array}\right]=\left[\begin{array}{c}
\left(\lambda I-A_{0}\right)^{-1}\left(B_{0} \int_{0}^{\infty} e^{-\lambda \eta} u(\eta) \mathrm{d} \eta+z\right) \\
\xi \mapsto-\int_{\xi}^{\infty} e^{\lambda(\xi-\eta)} u(\eta) \mathrm{d} \eta
\end{array}\right] \quad\left(\left[\begin{array}{l}
z \\
u
\end{array}\right] \in H \times \mathcal{U}_{\omega}\right), \\
& \left(\lambda I-\mathcal{C}_{\varepsilon}\right)^{-1}\left[\begin{array}{c}
z \\
u
\end{array}\right]=\left[\begin{array}{c}
\left(\lambda I-A_{\varepsilon}\right)^{-1}\left(B \int_{0}^{\infty} e^{-\lambda \eta} u(\eta) \mathrm{d} \eta+z\right) \\
\xi \mapsto-\int_{\xi}^{\infty} e^{\lambda(\xi-\eta)} u(\eta) \mathrm{d} \eta
\end{array}\right] \quad\left(\left[\begin{array}{l}
z \\
u
\end{array}\right] \in X \times \mathcal{U}_{\omega}\right)
\end{aligned}
$$

By combining Proposition 4.1, recalling that $B_{0}=P_{H} B$ and taking $z \in H$, it follows that

$$
\lim _{\varepsilon \rightarrow 0+}\left\|\left(\lambda I-\mathcal{C}_{\varepsilon}\right)^{-1}\left[\begin{array}{l}
z \\
u
\end{array}\right]-\left(\lambda I-\mathcal{C}_{0}\right)^{-1}\left[\begin{array}{l}
z \\
u
\end{array}\right]\right\|_{X \times \mathcal{U}}=0 \quad\left(\left[\begin{array}{c}
z \\
u
\end{array}\right] \in H \times \mathcal{U}_{\omega}\right) .
$$

Then the conclusion (4.26) follows from (4.34) and (4.25) by a slight variation of the Trotter-Kato theorem, in the spirit of the proof of Proposition 4.2. 
Remark 4.6. Note that for every $\omega>0, L^{2}([0, \infty) ; U)$ is contained in $\mathcal{U}_{\omega}$ so one can apply in particular Proposition 4.5 for controls $u \in L^{2}([0, \infty) ; U)$.

\subsection{Uniform constants in Datko's theorem}

The goal of this subsection is to provide a version of a well-known theorem of Datko, in which we make explicit the fact that the exponential decay rate of the semigroup depends only on the constants involved in assumptions (4.35) and (4.36) below.

Proposition 4.7. Let $\mathcal{A}$ be a generator of a strongly continuous semigroup $\left(\mathcal{F}_{t}\right)_{t \geqslant 0}$ on a Hilbert space $\mathcal{X}$. Assume that there exists a positive constant $C>0$ such that

$$
\left\|\mathcal{F}_{t}\right\|_{\mathcal{L}(X ; X)} \leqslant C e^{C t} \quad(t \geqslant 0)
$$

and

$$
\int_{0}^{\infty}\left\|\mathcal{F}_{t} h\right\|_{\mathcal{X}}^{2} \mathrm{~d} t \leqslant C\|h\|_{\mathcal{X}}^{2} \quad(h \in \mathcal{X})
$$

Then there exist $M>0$ and $\omega>0$, depending only on $C$, such that

$$
\left\|\mathcal{F}_{t}\right\|_{\mathcal{L}(\mathcal{X} ; \mathcal{X})} \leqslant M e^{-\omega t} \quad(t \geqslant 0)
$$

Proof. We closely follow the methodology used, for instance, in Tucsnak and Weiss ([32], Sect. 6.1), in proving Datko's theorem.

First, from (4.35), we have that for every $\tau>0$ there exists $m_{\tau} \geqslant 1$ (depending also on $C$ ) such that $\left\|\mathcal{F}_{t}\right\|_{\mathcal{L}(X ; X)} \leqslant m_{\tau}$ for all $t \in[0, \tau]$. Then

$$
\left\|\mathcal{F}_{\tau} h\right\|^{2}=\frac{1}{\tau} \int_{0}^{\tau}\left\|\mathcal{F}_{\tau-t} \mathcal{F}_{t} h\right\|^{2} \mathrm{~d} t \leqslant \frac{m_{\tau}^{2}}{\tau} \int_{0}^{\tau}\left\|\mathcal{F}_{t} h\right\|^{2} \mathrm{~d} t \quad(\tau>0, h \in \mathcal{X})
$$

By combining (4.38) and (4.36) it follows that

$$
c_{0}^{2}\|h\|^{2} \geqslant \sum_{k \in \mathbb{N}} \int_{(k-1) \tau}^{k \tau}\left\|\mathcal{F}_{t} h\right\|_{X}^{2} \mathrm{~d} t \geqslant \kappa_{\tau}^{2} \sum_{k \in \mathbb{N}}\left\|\mathcal{F}_{k \tau} h\right\|^{2} \quad(\tau>0, h \in \mathcal{X}),
$$

where $k_{\tau}=\frac{\sqrt{\tau}}{m_{\tau}}$. In particular, we see from the above that $\left\|\mathcal{F}_{k \tau}\right\|_{\mathcal{L}(\mathcal{X} ; \mathcal{X})} \leqslant \frac{c_{0}}{\kappa_{\tau}}$ for every $\tau>0$ and every $k \in \mathbb{N}$ (and this holds also for $k=0$ ). Hence, for every $\tau>0$, every $n \in \mathbb{N}$ and every $h \in \mathcal{X}$,

$$
\left\|\mathcal{F}_{n \tau} h\right\|^{2}=\frac{1}{n} \sum_{k=1}^{n}\left\|\mathcal{F}_{(n-k) \tau} \mathcal{F}_{k \tau} h\right\|^{2} \leqslant \frac{c_{0}^{2}}{n \kappa_{\tau}^{2}} \sum_{k=1}^{n}\left\|\mathcal{F}_{k \tau} h\right\|^{2} .
$$

By (4.39) we get that

$$
\left\|\mathcal{F}_{n \tau} h\right\|^{2} \leqslant \frac{c_{0}^{2}}{n \kappa_{\tau}^{2}} \cdot \frac{c_{0}^{2}}{\kappa_{\tau}^{2}}\|h\|^{2} \quad(\tau>0, n \in \mathbb{N}, h \in \mathcal{X})
$$


so that

$$
\left\|\mathcal{F}_{n \tau}\right\|_{\mathcal{L}(\mathcal{X} ; \mathcal{X})} \leqslant \frac{c_{0}^{2}}{\sqrt{n} \kappa_{\tau}^{2}} \quad(\tau>0, n \in \mathbb{N})
$$

Denoting $n_{\tau}=\left[\frac{4 c_{0}^{4}}{\kappa_{\tau}^{4}}\right]+1$ it follows that

$$
\inf _{n \in \mathbb{N}} \frac{1}{n \tau} \log \left\|\mathcal{F}_{n \tau}\right\|_{\mathcal{L}(\mathcal{X} ; \mathcal{X})} \leqslant \frac{1}{n_{\tau} \tau} \log \left\|\mathcal{F}_{n_{\tau} \tau}\right\|_{\mathcal{L}(\mathcal{X} ; \mathcal{X})} \leqslant \frac{-\log 2}{n_{\tau} \tau} \quad(\tau>0)
$$

Finally, combining the above estimate and a classical result (see, for instance, Tucsnak and Weiss [32], formula $(2.1 .3))$, it follows that

$$
\omega_{0}(\mathcal{F})=\inf _{t \in(0, \infty)} \frac{1}{t} \log \left\|\mathcal{F}_{t}\right\|_{\mathcal{L}(\mathcal{X} ; \mathcal{X})} \leqslant \inf _{n \in \mathbb{N}} \frac{1}{n \tau} \log \left\|\mathcal{F}_{n \tau}\right\|_{\mathcal{L}(\mathcal{X} ; \mathcal{X})} \leqslant \frac{-\log 2}{n_{\tau} \tau} \quad(\tau>0),
$$

which ends the proof.

\section{UNIFORM STABILIZABILITY AND NULL-CONTROLLABILITY RESULTS}

In the first part of this section we show that, assuming a smallness assumption on the stationary state $\left[\begin{array}{l}v_{s} \\ y_{s}\end{array}\right]$ (see Prop. 5.1), the semigroups $\mathbb{T}^{\varepsilon}$ generated by the operators $A_{\varepsilon}$ defined in (2.7) are uniformly (with respect to $\varepsilon$ ) exponentially stable. This will be a basic ingredient of the proof of Theorem 3.1. In the second part of this section we derive the corresponding ingredient of the proof of Theorem 3.2, which is is a uniform (again with respect to $\varepsilon$ ) null-controllability result for the pair $\left(A_{\varepsilon}, B\right)$, where $B$ is the operator defined in (3.9).

\subsection{Small stationary states and arbitrary control operator}

In this subsection we show that if the stationary state around which we linearized the problem is small (in an appropriate sense) then the semigroups $\left(\mathbb{T}^{\varepsilon}\right)_{\varepsilon>0}$ are uniformly exponentially stable. More precisely, we have:

Proposition 5.1. There exists $\delta>0$ such that if (3.3) holds then $A_{\varepsilon}$ generates a uniformly stable semigroup on $X$, i.e. there exist $M \geqslant 1$ and $\omega>0$ independent of $\varepsilon$ such that

$$
\left\|\mathbb{T}_{t}^{\varepsilon}\right\|_{\mathcal{L}(X ; X)} \leqslant M e^{-\omega t} \quad(\varepsilon>0, t \geqslant 0) .
$$

Proof. We split the proof into three steps.

Step 1. For every $\varepsilon>0$, consider the operator $\widetilde{A}_{\varepsilon}: \mathcal{D}\left(\widetilde{A}_{\varepsilon}\right)=\mathcal{D}\left(A_{\varepsilon}\right) \rightarrow X$ defined by

$$
\tilde{A}_{\varepsilon}\left[\begin{array}{l}
\varphi \\
\zeta
\end{array}\right]=\left[\begin{array}{c}
\nu \Delta \varphi+\frac{1}{\varepsilon} \nabla(\operatorname{div} \varphi)+\zeta e_{d} \\
\alpha \Delta \zeta
\end{array}\right] \quad\left(\left[\begin{array}{l}
\varphi \\
\zeta
\end{array}\right] \in \mathcal{D}\left(\widetilde{A}_{\varepsilon}\right)\right) .
$$

In other words, looking to (2.7), we have

$$
\tilde{A}_{\varepsilon}\left[\begin{array}{l}
\varphi \\
\zeta
\end{array}\right]=A_{\varepsilon}\left[\begin{array}{l}
\varphi \\
\zeta
\end{array}\right]+\left[\begin{array}{l}
\left(v_{s} \cdot \nabla\right) \varphi+(\varphi \cdot \nabla) v_{s} \\
\left(v_{s} \cdot \nabla \zeta\right)+\left(\varphi \cdot \nabla y_{s}\right)
\end{array}\right] \quad\left(\left[\begin{array}{c}
\varphi \\
\zeta
\end{array}\right] \in \mathcal{D}\left(\widetilde{A}_{\varepsilon}\right)\right)
$$


To show that the resolvent set of $\widetilde{A}_{\varepsilon}$ contains the half plane $\mathbb{C}_{-\beta}:=\{z \in \mathbb{C} ; \operatorname{Re} z>-\beta\}$ for some $\beta>0$, we note that the resolvent equation for $\widetilde{A}_{\varepsilon}$, that is

$$
\left(s I-\widetilde{A}_{\varepsilon}\right)\left[\begin{array}{l}
\varphi \\
\zeta
\end{array}\right]=\left[\begin{array}{l}
f \\
g
\end{array}\right] \in X
$$

can, according to $(2.8)$ and (5.2), be equivalently written as

$$
\begin{gathered}
s \int_{\Omega} \varphi \cdot \bar{\psi} \mathrm{d} x+\nu \int_{\Omega} \nabla \varphi: \nabla \bar{\psi} \mathrm{d} x+\frac{1}{\varepsilon} \int_{\Omega} \operatorname{div} \varphi \cdot \operatorname{div} \bar{\psi} \mathrm{d} x=\int_{\Omega} \zeta \cdot \overline{\psi_{d}} \mathrm{~d} x+\int_{\Omega} f \cdot \bar{\psi} \mathrm{d} x \\
s \int_{\Omega} \zeta \bar{\eta} \mathrm{d} x+\alpha \int_{\Omega} \nabla \zeta \cdot \nabla \bar{\eta} \mathrm{d} x=\int_{\Omega} g \bar{\eta} \mathrm{d} x,
\end{gathered}
$$

for every $\left[\begin{array}{l}\psi \\ \eta\end{array}\right] \in \mathcal{D}\left(A_{\varepsilon}\right)$. Note that the second equation is decoupled from the first. By Lax-Milgram's lemma and Poincaré's inequality, there exists $\beta_{1}>0$ such that for every $s \in \mathbb{C}_{-\beta_{1}},(5.5)$ admits a unique solution $\zeta=(s I-$ $\alpha \Delta)^{-1} g$. Inserting this information in (5.4), we see that by Lax-Milgram's lemma and Poincaré's inequality, there exists $\beta_{2}>0$ such that for every $s \in \mathbb{C}_{-\beta_{2}},(5.4)$ admits a unique solution $\varphi=\left(s I-\nu \Delta-\frac{1}{\varepsilon} \nabla(\operatorname{div})\right)^{-1}\left(\zeta_{d}+f\right)$. This proves that indeed the resolvent set of $\widetilde{A}_{\varepsilon}$ contains $\mathbb{C}_{-\beta}$ for $\beta=\min \left(\beta_{1}, \beta_{2}\right)>0$.

Step 2. We remark that there exists $\sigma \in(0, \beta)$ such that $\sigma I+\widetilde{A}_{\varepsilon}$ generates an exponentially stable analytic semigroup. Indeed, this is a direct application of Theorem 4.3 page 118 in [22], using Step 1 and Proposition 2.2. Step 3. We show that the operator $E$, with domain $\mathcal{D}(E)=H_{0}^{1}(\Omega)^{d+1}$, defined by

$$
\begin{aligned}
\left\langle E\left[\begin{array}{l}
\varphi \\
\zeta
\end{array}\right],\left[\begin{array}{l}
\psi \\
\eta
\end{array}\right]\right\rangle_{X}= & -\int_{\Omega}\left[\left(v_{s} \cdot \nabla\right) \varphi+(\varphi \cdot \nabla) v_{s}\right] \cdot \psi \mathrm{d} x \\
& -\int_{\Omega}\left[v_{s} \cdot \nabla \zeta+\varphi \cdot \nabla y_{s}\right] \cdot \bar{\eta} \mathrm{d} x \quad\left(\left[\begin{array}{c}
\varphi \\
\zeta
\end{array}\right],\left[\begin{array}{l}
\psi \\
\eta
\end{array}\right] \in \mathcal{D}(E)\right),
\end{aligned}
$$

is $\left(\sigma I+\widetilde{A}_{\varepsilon}\right)$-bounded in the usual sense, recalled below. To this aim, let $[\varphi, \psi] \in \mathcal{D}(E)$. We know from Step 1 , that $\left[\begin{array}{l}\varphi \\ \psi\end{array}\right]=\left(-\sigma I-\widetilde{A}_{\varepsilon}\right)^{-1}\left[\begin{array}{l}f \\ g\end{array}\right]$ for some $\left[\begin{array}{l}f \\ g\end{array}\right] \in X$ and there exists a constant $C>0$ such that

$$
\left\|\left[\begin{array}{l}
\varphi \\
\psi
\end{array}\right]\right\|_{H_{0}^{1}(\Omega)^{d+1}} \leqslant C\left\|\left[\begin{array}{l}
f \\
g
\end{array}\right]\right\|_{X}
$$

Assuming that (3.3) holds, we have by using (5.7) that

$$
\left\|E\left[\begin{array}{l}
\varphi \\
\psi
\end{array}\right]\right\|_{X} \leqslant \delta\left\|\left[\begin{array}{c}
\varphi \\
\psi
\end{array}\right]\right\|_{H_{0}^{1}(\Omega)^{d+1}} \leqslant C \delta\left\|\left[\begin{array}{l}
f \\
g
\end{array}\right]\right\|_{X} \leqslant C \delta\left\|\left(-\sigma I-\tilde{A}_{\varepsilon}\right)\left[\begin{array}{l}
\varphi \\
\psi
\end{array}\right]\right\|_{X} .
$$

Taking $\delta>0$ sufficiently small, and using Corollary 2.3, page 81 of [22] we obtain that $\tilde{A}_{\varepsilon}+\sigma I+E=A_{\varepsilon}+\sigma I$ generates a bounded analytic semigroup on $X$, so $A_{\varepsilon}$ generates a uniform stable semigroup on $X$ (note that $\sigma$ is independent of $\varepsilon>0$ ). This concludes the proof. 


\subsection{Possibly large stationary sates and controls distributed in a subdomain}

In the general case of a stationary state $\left[\begin{array}{l}v_{s} \\ y_{s}\end{array}\right] \in W^{1, \infty}(\Omega)^{d+1}$, with no smallness assumption, we cannot ensure that the operator $A_{\varepsilon}$ generates a stable semigroup, so we will use the control operator $B$ defined in (3.9) to stabilize this semigroup, uniformly with respect to (small enough) $\varepsilon$.

The main contribution in this subsection is the following uniform (with respect to $\varepsilon$ ) null controllability result.

Proposition 5.2. Let $\left(A_{\varepsilon}\right)_{\varepsilon>0}$ and $B$ be the operators introduced in (2.6), (2.7) and (3.9), respectively. Then for every $T>0$ there exist $\varepsilon_{0}>0$ and $C>0$ such that for every $\varepsilon \in\left(0, \varepsilon_{0}\right)$, the pair $\left(A_{\varepsilon}, B\right)$ is null controllable in time $T$ with control cost independent of $\varepsilon \in\left(0, \varepsilon_{0}\right)$. In other terms, for every $\left[\begin{array}{l}v_{0} \\ y_{0}\end{array}\right] \in X$, one can find a control $u_{\varepsilon}=\left[\begin{array}{c}\tilde{u}_{\varepsilon} \\ u_{d+1, \varepsilon}\end{array}\right] \in L^{2}((0, T) \times \mathcal{O})^{d+1}$ satisfying

$$
\left\|u_{\varepsilon}\right\|_{L^{2}((0, T) \times \mathcal{O})^{d+1}} \leqslant C\left\|\left[\begin{array}{c}
v_{0} \\
y_{0}
\end{array}\right]\right\|_{X},
$$

and such that the solution of (2.14) satisfies

$$
v_{\varepsilon}(T, \cdot)=0, \quad y_{\varepsilon}(T, \cdot)=0
$$

Remark 5.3. Using the standard duality between null controllability and finite state observability (see, for instance, [32], Thm. 11.2.1), Proposition 5.2 is a direct consequence of the following result:

Proposition 5.4. With the notation in Proposition 5.2, for every $T>0$ there exist $\varepsilon_{0}>0$ and $\kappa>0$ such that for every $\varepsilon \in\left(0, \varepsilon_{0}\right)$,

$$
\kappa^{2} \int_{0}^{T}\left\|B^{*}\left(\mathbb{T}_{t}^{\varepsilon}\right)^{*} \eta\right\|_{U}^{2} \mathrm{~d} t \geqslant\left\|\mathbb{T}_{T}^{\varepsilon *} \eta\right\|_{X}^{2} \quad(\eta \in X)
$$

where, for every $\varepsilon>0, \mathbb{T}^{\varepsilon *}$ is the analytic semigroup on $X$ generated by $A_{\varepsilon}^{*}$.

The remaining part of this subsection is essentially devoted to the proof of Proposition 5.4.

Remark 5.5. Recalling that the adjoint $A_{\varepsilon}^{*}$ is defined in $(4.2)$ and noticing that $B^{*} \in \mathcal{L}(X ; U)$ is given by

$$
B^{*}\left[\begin{array}{l}
\varphi \\
\zeta
\end{array}\right]=\left[\begin{array}{l}
\varphi \mathbb{1}_{\mathcal{O}} \\
\zeta \mathbb{1}_{\mathcal{O}}
\end{array}\right] \quad\left(\left[\begin{array}{l}
\varphi \\
\zeta
\end{array}\right] \in X\right)
$$

we see that the observability estimate (5.9) can be rephrased in PDE terms as follows: for every $\left[\begin{array}{c}\Phi_{0} \\ \Theta_{0}\end{array}\right] \in X$, the solution of

$$
\begin{cases}\partial_{t} \Phi-\nu \Delta \Phi-(\nabla \Phi)^{\operatorname{tr}} v_{s}-(\nabla \Phi) v_{s}+\Theta \nabla y_{s}+\nabla \Psi=0 & \text { in }(0, T) \times \Omega \\ \partial_{t} \Theta-\alpha \Delta \Theta-v_{s} \cdot \nabla \Theta=\Phi_{d} & \text { in }(0, T) \times \Omega, \\ \operatorname{div} \Phi+\varepsilon \Psi=0 & \text { in }(0, T) \times \Omega, \\ \Phi=0, \Theta=0 & \text { on }(0, T) \times \Gamma \\ \Phi(0, \cdot)=\Phi_{0}, \quad \Theta(0, \cdot)=\Theta_{0} & \text { in } \Omega,\end{cases}
$$


satisfies

$$
\left\|\left[\begin{array}{l}
\Phi \\
\Theta
\end{array}\right](T, \cdot)\right\|_{X}^{2} \leqslant \kappa^{2}\left\|\left[\begin{array}{c}
\Phi \\
\Theta
\end{array}\right]\right\|_{L^{2}((0, T) \times \mathcal{O})^{d+1}}^{2},
$$

where $\kappa$ is the constant in (5.9).

To prove (5.12), we will use Carleman estimates in the spirit of [1]. We have to introduce the classical weights of Carleman inequalities.

Let $\mathcal{O}_{0} \subset \subset \mathcal{O}$ and let $\eta \in C^{2}(\bar{\Omega})$ such that

$$
\begin{cases}\eta(x)>0 & (x \in \Omega) \\ \eta(x)=0 & (x \in \partial \Omega) \\ |\nabla \eta(x)|>0 & \left(x \in \overline{\Omega \backslash \mathcal{O}_{0}}\right)\end{cases}
$$

The existence of such a function is due to Imanuvilov, see Lemma 2.68 of [11] for a proof. Then, we introduce $l \in C^{\infty}([0, T])$ such that

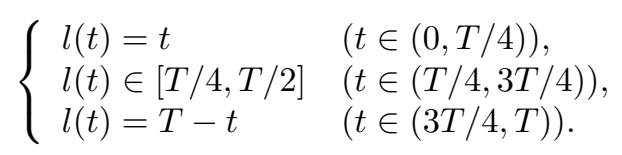

Finally, for $k \geqslant 2$ and $\lambda>1$, we define

$$
\alpha(t, x)=\frac{e^{2 \lambda\|\eta\|_{\infty}-e^{\lambda \eta(x)}}}{l(t)^{k}}, \quad \xi(t, x)=\frac{e^{\lambda\left(\eta(x)+\|\eta\|_{\infty}\right)}}{l(t)^{k}} \quad((t, x) \in[0, T] \times \Omega) .
$$

Setting $Q_{T}=(0, T) \times \Omega$, we also introduce the notation

$$
I(\lambda, s, \Theta)=s^{3} \lambda^{4} \int_{Q_{T}} e^{-2 s \alpha} \xi^{3}|\Theta|^{2} \mathrm{~d} t \mathrm{~d} x+s \lambda^{2} \int_{Q_{T}} e^{-2 s \alpha} \xi|\nabla \Theta|^{2} \mathrm{~d} t \mathrm{~d} x
$$

We are the now in a position to recall the standard Carleman estimate for the heat equation with homogeneous Dirichlet boundary conditions, see [13].

Proposition 5.6. There exists $\lambda_{0}>0$ such that for all $\lambda \geqslant \lambda_{0}$, there exist two constants $c_{0}(\lambda)$ and $s_{0}(\lambda)$ such that for all $s \geqslant s_{0}(\lambda)$, for every $\Theta_{0} \in L^{2}(\Omega), g \in L^{2}\left(Q_{T}\right)$ the solution $\Theta$ to

$$
\begin{cases}\partial_{t} \Theta-\alpha \Delta \Theta=g & \text { in }(0, T) \times \Omega \\ \Theta=0 & \text { on }(0, T) \times \Gamma \\ \Theta(0, \cdot)=\Theta_{0} & \text { in } \Omega\end{cases}
$$

satisfies

$$
I(\lambda, s, \Theta) \leqslant c_{0}(\lambda)\left(\int_{Q_{T}} e^{-2 s \alpha}|g|^{2} \mathrm{~d} t \mathrm{~d} x+s^{3} \lambda^{4} \int_{0}^{T} \int_{\mathcal{O}} e^{-2 s \alpha} \xi^{3}|\Theta|^{2} \mathrm{~d} t \mathrm{~d} x\right) .
$$


One of the main ingredient of the proof of (5.12) is a uniform Carleman estimate for the penalized Stokes system

$$
\begin{cases}\partial_{t} \Phi-\nu \Delta \Phi+\nabla \Psi=f & \text { in }(0, T) \times \Omega \\ \operatorname{div} \Phi+\varepsilon \Psi=0 & \text { in }(0, T) \times \Omega \\ \Phi=0 & \text { on }(0, T) \times \Gamma \\ \Phi(0, \cdot)=\Phi_{0} & \text { in } \Omega,\end{cases}
$$

with

$$
f=f_{0}+f_{1}+\sum_{j=1}^{n}\left(\left(\nabla f_{1, j}\right) z_{1, j}+\left(\nabla f_{2, j}\right)^{\mathrm{tr}} z_{2, j}\right), n \in \mathbb{N},
$$

where for $i=1,2$ and $j=1, \ldots, n$,

$$
\begin{array}{r}
f_{i} \in L^{2}\left(Q_{T}\right)^{d}, \nabla \times f_{1} \in L^{2}\left(Q_{T}\right)^{2 d-3}, \\
f_{i, j} \in L^{2}\left(0, T ;\left(H^{1}(\Omega)^{d}\right)\right), z_{i, j} \in L^{\infty}\left(0, T ;\left(W^{1, \infty}(\Omega)^{d}\right),\right.
\end{array}
$$

are given functions. In the previous equations, notation $\nabla \times f_{1}$ is used for the curl of $f_{1}$. To state this Carleman estimate we introduce the integral quantities:

$$
\begin{aligned}
I_{0}(\lambda, s, \Phi) & =s^{3} \lambda^{4} \int_{Q_{T}} e^{-2 s \alpha} \xi^{3}|\Phi|^{2} \mathrm{~d} t \mathrm{~d} x+s \lambda^{2} \int_{Q_{T}} e^{-2 s \alpha} \xi|\nabla \Phi|^{2} \mathrm{~d} t \mathrm{~d} x \\
& +s^{2} \lambda^{2} \int_{Q_{T}} e^{-2 s \alpha} \xi^{2}|\nabla \times \Phi|^{2} \mathrm{~d} t \mathrm{~d} x+\int_{Q_{T}} e^{-2 s \alpha}|\nabla(\nabla \times \Phi)|^{2} \mathrm{~d} t \mathrm{~d} x
\end{aligned}
$$

and

$$
\begin{aligned}
I_{1}(\lambda, s, f) & =s \int_{Q_{T}} e^{-2 s \alpha} \xi\left|f_{0}\right|^{2} \mathrm{~d} t \mathrm{~d} x \\
& +s^{1 / 2} \int_{Q_{T}} e^{-2 s \alpha} \xi\left|f_{1}\right|^{2} \mathrm{~d} t \mathrm{~d} x+s^{-1} \lambda^{-2} \int_{Q_{T}} e^{-2 s \alpha} \xi^{-1}\left|\nabla \times f_{1}\right|^{2} \mathrm{~d} t \mathrm{~d} x \\
& +\sum_{j=1}^{n} \sum_{i=1}^{2} \int_{Q_{T}} e^{-2 s \alpha}\left(s^{1 / 2} \xi\left|\nabla f_{i, j}\right|^{2}+s \xi\left|\nabla \times f_{i, j}\right|^{2}\right) \mathrm{d} t \mathrm{~d} x
\end{aligned}
$$

We have the following Carleman estimate, proved in Theorem 2.2 of [1].

Proposition 5.7. Let $\varepsilon_{0}>0$ and assume that $k \geqslant 4$. Then exists $\lambda_{0}>0$ such that for all $\lambda \geqslant \lambda_{0}$, there exist two constants $c_{0}(\lambda)$ and $s_{0}(\lambda)$ such that for every $s \geqslant s_{0}(\lambda)$ and all $\varepsilon \in\left(0, \varepsilon_{0}\right)$, for every $\Phi_{0} \in L^{2}(\Omega)^{d}$ and $f$ as in (5.17), the solution $\Phi$ to (5.16) satisfies

$$
I_{0}(\lambda, s, \Phi) \leqslant c_{0}(\lambda)\left(I_{1}(\lambda, s, f)+s^{4} \lambda^{4} \int_{0}^{T} \int_{\mathcal{O}} e^{-2 s \alpha} \xi^{3}|\Phi|^{2} \mathrm{~d} t \mathrm{~d} x\right) .
$$

Note that the constants $c_{0}(\lambda)$ and $s_{0}(\lambda)$ depend on $\left\|z_{i, j}\right\|_{L^{\infty}\left(0, T ; W^{1, \infty}(\Omega)^{d}\right)}$.

Putting together the two Carleman estimates above, stated. in Proposition 5.6 and Proposition 5.7 we obtain the following result: 
Proposition 5.8. Let $\varepsilon_{0}>0$ and assume that $k \geqslant 4$. There exists $\lambda_{0}>0$ such that for all $\lambda \geqslant \lambda_{0}$, there exist two constants $c_{0}(\lambda)$ and $s_{0}(\lambda)$ such that for every $s \geqslant s_{0}(\lambda)$ and all $\varepsilon \in\left(0, \varepsilon_{0}\right)$, for every $\left[\begin{array}{l}\Phi_{0} \\ \Theta_{0}\end{array}\right] \in X$, the solution $(\Phi, \Theta)$ to (5.11) satisfies

$$
I_{0}(\lambda, s, \Phi)+I(\lambda, s, \Theta) \leqslant c_{0}(\lambda)\left(s^{4} \lambda^{4} \int_{0}^{T} \int_{\mathcal{O}} e^{-2 s \alpha} \xi^{3}|\Phi|^{2} \mathrm{~d} t \mathrm{~d} x+s^{3} \lambda^{4} \int_{0}^{T} \int_{\mathcal{O}} e^{-2 s \alpha} \xi^{3}|\Theta|^{2} \mathrm{~d} t \mathrm{~d} x\right),
$$

with the constants $c_{0}(\lambda)$ and $s_{0}(\lambda)$ possibly depending on $\left\|\left[\begin{array}{l}v_{s} \\ y_{s}\end{array}\right]\right\|_{W^{1, \infty}(\Omega)^{d+1}}$.

Proof. In the following proof, the positive constants $C$ can vary from line to line and possibly depend on $\lambda$, $\left\|\left[\begin{array}{l}v_{s} \\ y_{s}\end{array}\right]\right\|_{W^{1, \infty}(\Omega)^{d+1}}$ but do not depend on $\varepsilon$.

First, by Proposition 5.7, we apply the Carleman estimate for the Stokes penalized system satisfied by $\Phi$ with $f$ as in (5.17) and

$$
n=1, f_{0}=-\Theta \nabla y_{s}, f_{1}=0, \quad f_{1,1}=f_{2,1}=\Phi, z_{1,1}=z_{2,1}=v_{s},
$$

then we obtain

$$
\begin{aligned}
I_{0}(\lambda, s, \Phi) \leqslant & C\left(s \int_{Q_{T}} e^{-2 s \alpha} \xi\left|f_{0}\right|^{2} \mathrm{~d} t \mathrm{~d} x\right. \\
& \left.+\sum_{j=1}^{n} \sum_{i=1}^{2} \int_{Q_{T}} e^{-2 s \alpha}\left(s^{1 / 2} \xi|\nabla y|^{2}+s \xi|\nabla \times y|^{2}\right) \mathrm{d} t \mathrm{~d} x+s^{4} \lambda^{4} \int_{0}^{T} \int_{\mathcal{O}} e^{-2 s \alpha} \xi^{3}|\Phi|^{2} \mathrm{~d} t \mathrm{~d} x\right) .
\end{aligned}
$$

Taking $s$ sufficiently large, according to the definition of $I_{0}$, we can absorb the second right hand side term in (5.20) to obtain

$$
I_{0}(\lambda, s, \Phi) \leqslant C\left(s \int_{Q_{T}} e^{-2 s \alpha} \xi|\Theta|^{2} \mathrm{~d} t \mathrm{~d} x+s^{4} \lambda^{4} \int_{0}^{T} \int_{\mathcal{O}} e^{-2 s \alpha} \xi^{3}|\Phi|^{2} \mathrm{~d} t \mathrm{~d} x\right) .
$$

We now use the Carleman estimate for the heat equation in Proposition 5.6 satisfied by $\Theta$ with $g=v_{s} \cdot \nabla \Theta+\Phi_{d}$ and we obtain

$$
I(\lambda, s, \Theta) \leqslant C\left(\int_{Q_{T}} e^{-2 s \alpha}\left(|\nabla \Theta|^{2}+|\Phi|^{2}\right) \mathrm{d} t \mathrm{~d} x+s^{3} \lambda^{4} \int_{0}^{T} \int_{\mathcal{O}} e^{-2 s \alpha} \xi^{3}|\Theta|^{2} \mathrm{~d} t \mathrm{~d} x\right) .
$$

We can absorb the first right hand side term in (5.22) taking $s$ sufficiently large to obtain

$$
I(\lambda, s, \Theta) \leqslant C\left(\int_{Q_{T}} e^{-2 s \alpha}|\Phi|^{2} \mathrm{~d} t \mathrm{~d} x+s^{3} \lambda^{4} \int_{0}^{T} \int_{\mathcal{O}} e^{-2 s \alpha} \xi^{3}|\Theta|^{2} \mathrm{~d} t \mathrm{~d} x\right) .
$$


We sum (5.21) and (5.23) to obtain

$$
\begin{aligned}
I_{0}(\lambda, s, \Phi)+I(\lambda, s, \Theta) & \leqslant C\left(s \int_{Q_{T}} e^{-2 s \alpha} \xi|\Theta|^{2} \mathrm{~d} t \mathrm{~d} x+\int_{Q_{T}} e^{-2 s \alpha}|\Phi|^{2} \mathrm{~d} t \mathrm{~d} x\right. \\
& \left.+s^{4} \lambda^{4} \int_{0}^{T} \int_{\mathcal{O}} e^{-2 s \alpha} \xi^{3}|\Phi|^{2} \mathrm{~d} t \mathrm{~d} x+s^{3} \lambda^{4} \int_{0}^{T} \int_{\mathcal{O}} e^{-2 s \alpha} \xi^{3}|\Theta|^{2} \mathrm{~d} t \mathrm{~d} x\right),
\end{aligned}
$$

and taking again $s$ sufficiently large, we can absorb the first and second right hand side terms of the above inequality to deduce (5.19).

Remark 5.9. The above proof can be adapted, by slightly changing the weights, to obtain that (5.19) holds without the term in the right hand side that containing $\Phi_{d}$. Indeed, by remarking that $\Phi_{d}=-\partial_{t} \Theta-\alpha \Delta \Theta-$ $v_{s} \cdot \nabla \Theta$, one can estimate the local term in $\Phi_{d}$ by a local term in $\Theta$ and global terms in $\Theta$. Moreover, these global terms can be absorbed by the left hand side of (5.19), see, for instance, Proof of Proposition 2.8 in [7] for the employment of such a technique. This actually leads to a null-controllability result as in Proposition 5.2 with a control of the form (7.1).

We are now in a position to prove the main result in this section.

Proof of Proposition 5.4. According to Remarks 5.3 and 5.5 it suffices to prove (5.12). The basic tool is the Carleman estimate (5.19) above. Let $\lambda$ and $s$ be sufficiently large such that (5.19) holds. We first remark that there exist $c_{1}, c_{2}>0$ such that

$$
\begin{gathered}
s^{3} \lambda^{4} e^{-2 s \alpha} \xi^{3} \geqslant c_{1} \quad(t \in(T / 4,3 T / 4), x \in \Omega) . \\
s^{4} \lambda^{4} e^{-2 s \alpha} \xi^{3}+s^{3} \lambda^{4} e^{-2 s \alpha} \xi^{3} \leqslant c_{2} \quad(t \in(0, T), x \in \mathcal{O}) .
\end{gathered}
$$

Using (5.19), (5.24) and (5.25) we have that

$$
\int_{T / 4}^{3 T / 4} \int_{\Omega}\left(|\Phi|^{2}+|\Theta|^{2}\right) \mathrm{d} t \mathrm{~d} x \leqslant c_{3} \int_{0}^{T} \int_{\mathcal{O}}\left(|\Phi|^{2}+|\Theta|^{2}\right) \mathrm{d} t \mathrm{~d} x,
$$

for some constant $c_{3}>0$. By using (4.9), i.e. the semigroups $\left(\left(\mathbb{T}^{\varepsilon}\right)^{*}\right)_{\varepsilon>0}$ have a growth bound independent of $\varepsilon>0$, it follows that there exists $c_{4}>0$ such that

$$
\left\|\left[\begin{array}{c}
\Phi \\
\Theta
\end{array}\right](T, \cdot)\right\|_{X}^{2} \leqslant c_{4} \int_{T / 4}^{3 T / 4}\left\|\left[\begin{array}{c}
\Phi \\
\Theta
\end{array}\right](t, \cdot)\right\|_{X}^{2} \mathrm{~d} t .
$$

Gathering (5.26) and (5.27) we deduce the expected observability estimate (5.12).

Remark 5.10. For obtaining the null-controllability result of Proposition 5.2 for the pair $\left(A_{0}, B_{0}\right)$, one can follow exactly the same steps that we have done, using Lemma 3.1 of [13] for Carleman estimates applied to the Stokes system.

\section{Proof of the MAIN Results}

The goal of this section is to prove Theorem 3.1 and Theorem 3.2.

We first use Proposition 5.1 to prove (3.4) and (3.5) for Theorem 3.1. 
Proofs of (3.4) and (3.5) for Theorem 3.1. We have from Theorem 2.5 and Proposition 5.1 that

$$
\left\langle\Pi_{\varepsilon}\left[\begin{array}{l}
v_{0} \\
y_{0}
\end{array}\right],\left[\begin{array}{l}
v_{0} \\
y_{0}
\end{array}\right]\right\rangle \leqslant J\left(0 ;\left[\begin{array}{l}
v_{0} \\
y_{0}
\end{array}\right]\right) \leqslant \int_{0}^{+\infty}\left\|\mathbb{T}_{t}^{\varepsilon}\left[\begin{array}{l}
v_{0} \\
y_{0}
\end{array}\right]\right\|_{X}^{2} \mathrm{~d} t \leqslant C\left\|\left[\begin{array}{l}
v_{0} \\
y_{0}
\end{array}\right]\right\|_{X}^{2}\left(\varepsilon>0,\left[\begin{array}{l}
v_{0} \\
y_{0}
\end{array}\right] \in X\right),
$$

which implies (3.4). Moreover,

$$
\int_{0}^{+\infty}\left\|\mathbb{F}_{t}^{\varepsilon}\left[\begin{array}{c}
v_{0} \\
y_{0}
\end{array}\right]\right\|_{X}^{2} \mathrm{~d} t \leqslant J\left(0 ;\left[\begin{array}{c}
v_{0} \\
y_{0}
\end{array}\right]\right) \leqslant \int_{0}^{+\infty}\left\|\mathbb{T}_{t}^{\varepsilon}\left[\begin{array}{l}
v_{0} \\
y_{0}
\end{array}\right]\right\|_{X}^{2} \mathrm{~d} t \leqslant C\left\|\left[\begin{array}{l}
v_{0} \\
y_{0}
\end{array}\right]\right\|_{X}^{2}\left(\varepsilon>0,\left[\begin{array}{l}
v_{0} \\
y_{0}
\end{array}\right] \in X\right),
$$

which implies (3.5) by using Proposition 4.7.

We secondly use Proposition 5.2 to prove assertions (3.4) and (3.5) in Theorem 3.2.

Proof of (3.4) and (3.5) in Theorem 3.2. From Proposition 5.2, by fixing some $T>0$, we know that there exists $\varepsilon_{0}>0$ such that for every $\varepsilon \in\left(0, \varepsilon_{0}\right)$ and every $\left[\begin{array}{l}v_{0} \\ y_{0}\end{array}\right] \in X$, one can find a control $u_{\varepsilon} \in L^{2}((0, T) \times \mathcal{O})^{d+1}$ satisfying (5.8) and such that the associated solution of $(2.14)$ satisfies $v_{\varepsilon}(T, \cdot)=0$ and $y_{\varepsilon}(T, \cdot)=0$. By extending the control $u_{\varepsilon}$ by 0 on $(T,+\infty) \times \mathcal{O}$, the associated solution satisfies $v_{\varepsilon}(t, \cdot)=0$ and $y_{\varepsilon}(t, \cdot)=0$ for every $t \geqslant T$. Moreover, using (2.9), (2.15) and (5.8), we obtain that there exists a constant $C>0$ independent of $\varepsilon$ such that

$$
\left\|\left[\begin{array}{l}
v_{\varepsilon} \\
y_{\varepsilon}
\end{array}\right]\right\|_{C([0, T] ; X)} \leqslant C\left\|\left[\begin{array}{l}
v_{0} \\
y_{0}
\end{array}\right]\right\|_{X} \quad\left(\varepsilon \in\left(0, \varepsilon_{0}\right),\left[\begin{array}{l}
v_{0} \\
y_{0}
\end{array}\right] \in X\right) .
$$

We can thus combine Theorem 2.5, (5.8) and (6.1) to get

$$
\begin{aligned}
& \left\langle\Pi_{\varepsilon}\left[\begin{array}{l}
v_{0} \\
y_{0}
\end{array}\right],\left[\begin{array}{l}
v_{0} \\
y_{0}
\end{array}\right]\right\rangle \leqslant C J\left(u_{\varepsilon} ;\left[\begin{array}{l}
v_{0} \\
y_{0}
\end{array}\right]\right) \leqslant \int_{0}^{T}\left(\left\|u_{\varepsilon}(t)\right\|_{L^{2}(\mathcal{O})^{d+1}}^{2}+\left\|\left[\begin{array}{l}
v_{\varepsilon}(t) \\
y_{\varepsilon}(t)
\end{array}\right]\right\|_{X}^{2}\right) \mathrm{d} t \\
& \leqslant C\left\|\left[\begin{array}{l}
v_{0} \\
y_{0}
\end{array}\right]\right\|_{X}^{2} \quad\left(\varepsilon \in\left(0, \varepsilon_{0}\right),\left[\begin{array}{l}
v_{0} \\
y_{0}
\end{array}\right] \in X\right) .
\end{aligned}
$$

The above estimate implies (3.4) for $\varepsilon \in\left(0, \varepsilon_{0}\right)$. Moreover, we have

$$
\begin{aligned}
& \int_{0}^{+\infty}\left\|\mathbb{F}_{t}^{\varepsilon}\left[\begin{array}{l}
v_{0} \\
y_{0}
\end{array}\right]\right\|_{X}^{2} \mathrm{~d} t \leqslant C J\left(u_{\varepsilon} ;\left[\begin{array}{l}
v_{0} \\
y_{0}
\end{array}\right]\right) \leqslant \int_{0}^{T}\left(\left\|u_{\varepsilon}(t)\right\|_{L^{2}(\mathcal{O})^{d+1}}^{2}+\left\|\left[\begin{array}{l}
v_{\varepsilon}(t) \\
y_{\varepsilon}(t)
\end{array}\right]\right\|_{X}^{2}\right) \mathrm{d} t \\
& \leqslant C\left\|\left[\begin{array}{l}
v_{0} \\
y_{0}
\end{array}\right]\right\|_{X}^{2} \quad\left(\varepsilon \in\left(0, \varepsilon_{0}\right),\left[\begin{array}{l}
v_{0} \\
y_{0}
\end{array}\right] \in X\right) .
\end{aligned}
$$

which can be combined with Proposition 4.7 to yield (3.5) for $\varepsilon \in\left(0, \varepsilon_{0}\right)$.

For the sake of simplicity, we omit below, while using (3.4) and (3.5), the condition $\varepsilon \in\left(0, \varepsilon_{0}\right)$, which will be often replaced $\varepsilon \in\left(0, \varepsilon_{0}\right)$ (as in Thm. 3.2).

In order to complete the proofs of Theorem 3.1 and Theorem 3.2 we still need to check the convergence results stated in (3.6), (3.7) and (3.8). This will be done by first proving that similar convergence properties hold for the corresponding finite horizon LQR problems, in the spirit of Appendix in [5]. To this aim, we first recall some general results concerning this type of approximation.

Let $\mathcal{A}$ be a generator of a strongly continuous semigroup $\left(\mathcal{T}_{t}\right)_{t \geqslant 0}$ on a Hilbert space $\mathcal{X}$, let $\mathcal{B} \in \mathcal{L}(\mathcal{U} ; \mathcal{X})$ be the (bounded) control operator where the input space $\mathcal{U}$ is also a Hilbert space. Consider regulator problems 
on the finite intervals $\left[s, t_{f}\right]$ with $-\infty<s<t_{f}$, with a weighting symmetric bounded positive operator $G \geqslant 0$ for the final state $x\left(t_{f}\right)$. The finite interval problems are given by

$$
\begin{gathered}
\left(\mathcal{R}, t_{f}\right): \text { Minimize } J(u ; s, x(s))=\left\langle G x\left(t_{f}\right), x\left(t_{f}\right)\right\rangle+\int_{s}^{t_{f}}\left(\|x(t)\|_{\mathcal{X}}^{2}+\|u(t)\|_{\mathcal{U}}^{2}\right) \mathrm{d} t \\
\text { subject to } x(t)=\mathcal{T}_{t} x_{s}+\int_{s}^{t} \mathcal{T}_{t-\sigma} B u(\sigma) d \sigma, s \leqslant t \leqslant t_{f} .
\end{gathered}
$$

In the above context it is known that a unique nonnegative selfadoint Riccati operator function $\Pi_{s}$ can be associated with $\left(\mathcal{R}, t_{f}\right)$, see, for instance, Theorem 3.2 of [15]. More precisely, $\Pi_{s}$ satisfies the integral Riccati equation

$$
\Pi_{s}(t) x=\mathcal{T}_{t_{f}-f}^{*} G \mathcal{T}_{t_{f}-t} x+\int_{t_{f}}^{t} \mathcal{T}_{\eta-t}^{*}\left(I-\Pi_{s}(\eta) B^{*} \Pi_{s}(\eta)\right) \mathcal{T}_{\eta-t} x \mathrm{~d} \eta \quad\left(x \in \mathcal{X}, \in\left[s, t_{f}\right]\right)
$$

with $\Pi_{s}(\xi) \in \mathcal{L}(\mathcal{X})$ a self-adjoint operator for every $\xi \in\left[s, t_{f}\right]$.

We have the following classical result, see, for instance, Theorem A.1 of [5]:

Theorem 6.1. Assume that the unique nonnegative selfadjoint solution $\Pi$ of the Riccati equation (2.18) exists. Let $\Pi_{s}$ be the unique Riccati operator function associated with the problem $\left(\mathcal{R}, t_{f}=0\right)$. If $\lim _{t \rightarrow \infty}\left\|\mathcal{F}_{t} x_{0}\right\|=0$ for all $x_{0} \in \mathcal{X}$, where $\mathcal{F}:=\left(\mathcal{F}_{t}\right)_{t \geqslant 0}$ is the closed loop semigroup, i.e., the one generated by $\mathcal{A}-\mathcal{B} \mathcal{B}^{*} \Pi$, then we have

$$
\lim _{s \rightarrow-\infty} \Pi_{s}(s) x_{0}=\Pi x_{0} \quad\left(x_{0} \in \mathcal{X}\right)
$$

If moreover $G \geqslant \Pi$ (in the sense of symmetric operators) and there exist positive constants $M$ and $\beta$ such that

$$
\left\|\mathcal{F}_{t}\right\| \leqslant M e^{-\beta t} \quad(t \geqslant 0)
$$

then

$$
\Pi \leqslant \Pi_{s}(s) \leqslant \Pi+M^{2} e^{2 \beta s}\|G\| \quad(s<0) .
$$

By coming back to our situation, calling $\left(\mathcal{R}^{\varepsilon}, t_{f}=0\right)$ the finite horizon regulator problem associated to $\left(A_{\varepsilon}, B\right)$, and $\left(\mathcal{R}^{0}, t_{f}=0\right)$ the finite horizon regulator problem associated to $\left(A_{0}, B_{0}\right)$, we can show the following result, which is an adaptation of Theorem 5.1 in [15] to our context.

Theorem 6.2. For every $s<0$, for every $\left[\begin{array}{l}v_{0} \\ y_{0}\end{array}\right] \in H$, we have

$$
\begin{array}{r}
\lim _{\varepsilon \rightarrow 0}\left\|u_{\mathrm{opt}, \varepsilon}(t)-u_{\mathrm{opt}}(t)\right\|_{U}=0, \\
\lim _{\varepsilon \rightarrow 0}\left\|\left[\begin{array}{l}
v_{\mathrm{opt}, \varepsilon}(t) \\
y_{\mathrm{opt}, \varepsilon}(t)
\end{array}\right]-\left[\begin{array}{l}
v_{\mathrm{opt}}(t) \\
y_{\mathrm{opt}}(t)
\end{array}\right]\right\|_{X}=0, \\
\lim _{\varepsilon \rightarrow 0}\left\|\Pi_{s, \varepsilon}(t)\left[\begin{array}{l}
v_{0} \\
y_{0}
\end{array}\right]-\Pi_{s}(t)\left[\begin{array}{l}
v_{0} \\
y_{0}
\end{array}\right]\right\|_{X}=0,
\end{array}
$$

uniformly in $t \in[s, 0]$. Here, $u_{\mathrm{opt}, \varepsilon}, u_{\mathrm{opt}},\left[\begin{array}{l}v_{\mathrm{opt}, \varepsilon} \\ y_{\mathrm{opt}, \varepsilon}\end{array}\right],\left[\begin{array}{l}v_{\mathrm{opt}} \\ y_{\mathrm{opt}}\end{array}\right]$ denote respective optimal controls and trajectories of the problems $\left(\mathcal{R}^{\varepsilon}, 0\right)$ associated to $\left(A_{\varepsilon}, B\right)$ and $\left(\mathcal{R}^{0}, 0\right)$ associated to $\left(A_{0}, B\right)$. 
Proof. Take $s<0, \tau \geqslant s$ and $\left[\begin{array}{l}v_{0} \\ y_{0}\end{array}\right] \in H$. First, we remark that we just have to prove (6.6). Indeed, assuming that we have (6.6), the optimal trajectory of the penalized problem is given by

$$
\mathbb{S}_{t-\tau}^{\varepsilon}\left[\begin{array}{l}
v_{0} \\
y_{0}
\end{array}\right]:=\left[\begin{array}{l}
v_{\mathrm{opt}, \varepsilon}(t) \\
y_{\mathrm{opt}, \varepsilon}(t)
\end{array}\right]=\mathbb{T}_{t-\tau}^{\varepsilon}\left[\begin{array}{l}
v_{0} \\
y_{0}
\end{array}\right]-\int_{\tau}^{t} \mathbb{T}_{t-\eta}^{\varepsilon} B u_{\mathrm{opt}, \varepsilon}(\eta) \mathrm{d} \eta \quad(\varepsilon>0, \tau \leqslant t \leqslant 0) .
$$

By Proposition 4.2, we have

$$
\lim _{\varepsilon \rightarrow 0}\left\|\mathbb{T}_{t-\tau}^{\varepsilon}\left[\begin{array}{l}
v_{0} \\
y_{0}
\end{array}\right]-\mathbb{T}_{t-\tau}^{0}\left[\begin{array}{l}
v_{0} \\
y_{0}
\end{array}\right]\right\|_{X}=0 \quad \text { uniformly in } t \in[\tau, 0]
$$

For the second term in the right hand side of (6.9), we write

$$
\begin{gathered}
\int_{\tau}^{t} \mathbb{T}_{t-\eta}^{\varepsilon} B u_{\mathrm{opt}, \varepsilon}(\eta) \mathrm{d} \eta-\int_{\tau}^{t} \mathbb{T}_{t-\eta}^{0} B_{0} u_{\mathrm{opt}}(\eta) \mathrm{d} \eta=\int_{\tau}^{t} \mathbb{T}_{t-\eta}^{\varepsilon} B u_{\mathrm{opt}, \varepsilon}(\eta) \mathrm{d} \eta-\int_{\tau}^{t} \mathbb{T}_{t-\eta}^{\varepsilon} B u_{\mathrm{opt}}(\eta) \mathrm{d} \eta \\
+\int_{\tau}^{t} \mathbb{T}_{t-\eta}^{\varepsilon} B u_{\mathrm{opt}}(\eta) \mathrm{d} \eta-\int_{\tau}^{t} \mathbb{T}_{t-\eta}^{0} B u_{\mathrm{opt}}(\eta) \mathrm{d} \eta \quad(\varepsilon>0, \tau \leqslant t \leqslant 0) .
\end{gathered}
$$

By using (6.6) and the growth bound of $\mathbb{T}^{\varepsilon}$ given in (2.9), we get that the first right hand side term of the above formula goes to 0 as $\varepsilon \rightarrow 0$, uniformly in $t \in[\tau, 0]$. Next, by using the convergence of Lax-Phillips semigroup, given in Proposition 4.5, we obtain that the second right hand side term of the above formula also goes to 0 as $\varepsilon \rightarrow 0$, uniformly in $t \in[\tau, 0]$. This yields

$$
\lim _{\varepsilon \rightarrow 0}\left\|\int_{\tau}^{t} \mathbb{T}_{t-\eta}^{\varepsilon} B u_{\mathrm{opt}, \varepsilon}(\eta) \mathrm{d} \eta-\int_{\tau}^{t} \mathbb{T}_{t-\eta}^{0} B_{0} u_{\mathrm{opt}}(\eta) \mathrm{d} \eta\right\|_{X}=0 \quad \text { uniformly in } t \in[\tau, 0] .
$$

By gathering (6.9), (6.10) and (6.11), we obtain (6.7).

Moreover, we have by Equation (3.25) in [15],

$$
\Pi_{s, \varepsilon}(t)\left[\begin{array}{l}
v_{0} \\
y_{0}
\end{array}\right]=\left(\mathbb{T}_{t_{f}-t}^{\varepsilon}\right)^{*} G \mathbb{S}_{t_{f}-t}^{\varepsilon}\left[\begin{array}{l}
v_{0} \\
y_{0}
\end{array}\right]+\int_{t}^{t_{f}}\left(\mathbb{T}_{\eta-t}^{\varepsilon}\right)^{*} \mathbb{S}_{\eta-t}^{\varepsilon}\left[\begin{array}{l}
v_{0} \\
y_{0}
\end{array}\right] \mathrm{d} \eta, \quad(\varepsilon>0, \tau \leqslant t \leqslant 0),
$$

so we can readily pass to the limit as $\varepsilon \rightarrow 0$, in the above formula, using Proposition 4.2 and (6.7). This leads to $(6.8)$.

According to Equation (3.14) in [15], we have

$$
u_{\mathrm{opt}, \varepsilon}(t)=-\left(\tilde{Q}_{s}^{\varepsilon}\right)^{-1}\left(\tilde{B}_{s}^{\varepsilon *}\left[\begin{array}{c}
v_{0} \\
y_{0}
\end{array}\right]\right)(t) \quad(\varepsilon>0, \tau \leqslant t \leqslant 0),
$$

where $\tilde{Q}_{s}^{\varepsilon}$ and $\tilde{B}_{s}^{\varepsilon}$ are (linear) bounded operators defined in equations (3.10), (3.11) of [15]. By following line by line ([15], Eqs. (5.8)-(5.11)), using Proposition 4.2, we obtain the expected convergence (6.6).

We are now in a position to prove (3.6), (3.7) and (3.8) for both Theorem 3.1 and Theorem 3.2, recalling that we already know that $\left(A_{0}, B_{0}\right)$ is stabilizable and that we have already checked (3.4) and (3.5).

Proofs of (3.6), (3.7) and (3.8). We first prove (3.6). We take $G=\tilde{M} I_{d}$ where $\tilde{M}$ corresponds to the uniform bound for the Riccati operator $\Pi_{\varepsilon}$ in (3.4). We apply Theorem 6.1 to each problem $\left(\mathcal{R}^{\varepsilon}, 0\right)$ on $X$ and by using 
the uniform bound for the feedback semigroup, stated in (3.5), we conclude that for $s<0$, we have

$$
\Pi_{\varepsilon} \leqslant \Pi_{s, \varepsilon}(s) \leqslant \Pi_{\varepsilon}+M^{2} e^{2 \omega s} \tilde{M} .
$$

Let us fix $\left[\begin{array}{l}v_{0} \\ y_{0}\end{array}\right] \in H \backslash\{0\}$ and $N \in \mathbb{N}^{*}$. From (6.13), we know that there exists $C_{0}>0$ such that

$$
\left\|\Pi_{\zeta, \varepsilon}(\zeta)-\Pi_{\varepsilon}\right\|_{\mathcal{L}(X ; X)} \leqslant \frac{1}{4 N\left\|\left[\begin{array}{l}
v_{0} \\
y_{0}
\end{array}\right]\right\|_{X}} \quad\left(\varepsilon>0, \zeta<-C_{0}\right)
$$

Using the fact that $\left(A_{0}, B_{0}\right)$ is stabilizable, we have that (6.3) holds for $\Pi$ (here $\Pi$ actually denotes $\Pi_{0}$ to avoid confusion with $\Pi_{s}$ or $\left.\Pi_{\zeta}\right)$. From Theorem 6.1 applied to the problem $\left(\mathcal{R}^{0}, 0\right)$ on $H$, we then know that there exists $C_{1}>0$ such that

$$
\left\|\Pi_{\zeta}(\zeta)-\Pi\right\|_{\mathcal{L}(X ; X)} \leqslant \frac{1}{4 N\left\|\left[\begin{array}{l}
v_{0} \\
y_{0}
\end{array}\right]\right\|_{X}} \quad\left(\zeta<-C_{1}\right) .
$$

Therefore, from the triangle inequality, we deduce that for $\zeta<-\max \left(C_{0}, C_{1}\right)$,

$$
\begin{aligned}
\left\|\Pi_{\varepsilon}\left[\begin{array}{c}
v_{0} \\
y_{0}
\end{array}\right]-\Pi\left[\begin{array}{c}
v_{0} \\
y_{0}
\end{array}\right]\right\|_{X} & \leqslant\left\|\Pi_{\varepsilon}-\Pi_{\zeta, \varepsilon}(\zeta)\right\|_{\mathcal{L}(X ; X)}\left\|\left[\begin{array}{c}
v_{0} \\
y_{0}
\end{array}\right]\right\|_{X}+\left\|\Pi_{\zeta, \varepsilon}(\zeta)\left[\begin{array}{c}
v_{0} \\
y_{0}
\end{array}\right]-\Pi_{\zeta}(\zeta)\left[\begin{array}{c}
v_{0} \\
y_{0}
\end{array}\right]\right\|_{X} \\
& +\left\|\Pi_{\zeta}-\Pi\right\|_{\mathcal{L}(X ; X)}\left\|\left[\begin{array}{c}
v_{0} \\
y_{0}
\end{array}\right]\right\|_{X} \\
& \leqslant \frac{1}{2 N}+\left\|\Pi_{\zeta, \varepsilon}(\zeta)\left[\begin{array}{c}
v_{0} \\
y_{0}
\end{array}\right]-\Pi_{\zeta}(\zeta)\left[\begin{array}{c}
v_{0} \\
y_{0}
\end{array}\right]\right\|_{X} \quad(\varepsilon>0) .
\end{aligned}
$$

We know from Theorem 6.2 that the second term in right hand side term of the above equation goes to 0 as $\varepsilon \rightarrow 0$, so there exists $\varepsilon^{\prime}$ such that for every $\varepsilon \in\left(0, \varepsilon^{\prime}\right)$, we obtain

$$
\left\|\Pi_{\varepsilon}\left[\begin{array}{l}
v_{0} \\
y_{0}
\end{array}\right]-\Pi\left[\begin{array}{l}
v_{0} \\
y_{0}
\end{array}\right]\right\|_{X} \leqslant \frac{1}{2 N}+\frac{1}{2 N} \leqslant \frac{1}{N}
$$

which, recalling that $N \in \mathbb{N}^{*}$ is arbitrary, concludes the proof of (3.6).

We next prove the convergence of the feedback operators, i.e. (3.7). To this aim we first note that for every $\left[\begin{array}{l}v_{0} \\ y_{0}\end{array}\right] \in H$ we have

$$
\begin{aligned}
\mathbb{F}_{t}^{\varepsilon}\left[\begin{array}{l}
v_{0} \\
y_{0}
\end{array}\right]-\mathbb{F}_{t}^{0}\left[\begin{array}{l}
v_{0} \\
y_{0}
\end{array}\right]=\mathbb{T}_{t}^{\varepsilon}\left[\begin{array}{l}
v_{0} \\
y_{0}
\end{array}\right]-\mathbb{T}_{t}^{0}\left[\begin{array}{l}
v_{0} \\
y_{0}
\end{array}\right] \\
+\int_{0}^{t} \mathbb{T}_{t-\eta}^{\varepsilon}\left(-B B^{*} \Pi_{\varepsilon} \mathbb{F}_{\eta}^{\varepsilon}\right)\left[\begin{array}{l}
v_{0} \\
y_{0}
\end{array}\right] \mathrm{d} \eta-\int_{0}^{t} \mathbb{T}_{t-\eta}^{0}\left(-B_{0} B_{0}^{*} \Pi_{0} \mathbb{F}_{\eta}^{0}\right)\left[\begin{array}{l}
v_{0} \\
y_{0}
\end{array}\right] \mathrm{d} \eta \quad(\varepsilon>0, t \geqslant 0) .
\end{aligned}
$$

The quantity defined by the first two terms in the right hand side of (6.17) tends to 0 as $\varepsilon \rightarrow 0$, uniformly on compact intervals in time. For the remaining terms in the right hand side we write 


$$
\begin{aligned}
\int_{0}^{t}\left[\mathbb{T}_{t-\eta}^{\varepsilon}(\right. & \left.\left.-B B^{*} \Pi_{\varepsilon} \mathbb{F}_{\eta}^{\varepsilon}\right)-\mathbb{T}_{t-\eta}^{0}\left(-B_{0} B_{0}^{*} \Pi_{0} \mathbb{F}_{\eta}^{0}\right)\right]\left[\begin{array}{l}
v_{0} \\
y_{0}
\end{array}\right] \mathrm{d} \eta \\
= & \int_{0}^{t}\left[\mathbb{T}_{t-\eta}^{\varepsilon}\left(-B B^{*} \Pi_{\varepsilon} \mathbb{F}_{\eta}^{\varepsilon}\right)-\mathbb{T}_{t-\eta}^{\varepsilon}\left(-B B^{*} \Pi_{\varepsilon} \mathbb{F}_{\eta}^{0}\right)+\mathbb{T}_{t-\eta}^{\varepsilon}\left(-B B^{*} \Pi_{\varepsilon} \mathbb{F}_{\eta}^{0}\right)-\mathbb{T}_{t-\eta}^{\varepsilon}\left(-B B^{*} \Pi_{0} \mathbb{F}_{\eta}^{0}\right)\right. \\
& \left.+\mathbb{T}_{t-\eta}^{\varepsilon}\left(-B B_{0}^{*} \Pi_{0} \mathbb{F}_{\eta}^{0}\right)-\mathbb{T}_{t-\eta}^{0}\left(-B_{0} B_{0}^{*} \Pi_{0} \mathbb{F}_{\eta}^{0}\right)\right]\left[\begin{array}{c}
v_{0} \\
y_{0}
\end{array}\right] \mathrm{d} \eta \quad(\varepsilon>0, t \geqslant 0) .
\end{aligned}
$$

Consequently, from (6.17), (6.18) and the uniform bound (2.9), we obtain that taking $T>0$, there exists a constant $C>0$ such that for every $\varepsilon>0$ and $t \in[0, T]$,

$$
\begin{aligned}
\| \mathbb{F}_{t}^{\varepsilon}\left[\begin{array}{l}
v_{0} \\
y_{0}
\end{array}\right]- & \mathbb{F}_{t}^{0}\left[\begin{array}{l}
v_{0} \\
y_{0}
\end{array}\right]\left\|_{X} \leqslant\right\| \mathbb{T}_{t}^{\varepsilon}\left[\begin{array}{l}
v_{0} \\
y_{0}
\end{array}\right]-\mathbb{T}_{t}^{0}\left[\begin{array}{l}
v_{0} \\
y_{0}
\end{array}\right]\left\|_{X}+C \int_{0}^{t}\right\| \Pi_{\varepsilon} \mathbb{F}_{\eta}^{0}\left[\begin{array}{l}
v_{0} \\
y_{0}
\end{array}\right]-\Pi_{\eta}^{0} \mathbb{F}_{\eta}^{0}\left[\begin{array}{l}
v_{0} \\
y_{0}
\end{array}\right] \|_{X} \mathrm{~d} \eta \\
& +\int_{0}^{t}\left\|\mathbb{T}_{t-\eta}^{\varepsilon}\left(-B B_{0}^{*} \Pi_{0} \mathbb{F}_{\eta}^{0}\right)\left[\begin{array}{l}
v_{0} \\
y_{0}
\end{array}\right]-\mathbb{T}_{t-\eta}^{0}\left(-B_{0} B_{0}^{*} \Pi_{0} \mathbb{F}_{\eta}^{0}\right)\left[\begin{array}{c}
v_{0} \\
y_{0}
\end{array}\right]\right\|_{X} \mathrm{~d} \eta+C \int_{0}^{t}\left\|\mathbb{F}_{\eta}^{\varepsilon}\left[\begin{array}{c}
v_{0} \\
y_{0}
\end{array}\right]-\mathbb{F}_{\eta}^{0}\left[\begin{array}{c}
v_{0} \\
y_{0}
\end{array}\right]\right\|_{X} \mathrm{~d} \eta .
\end{aligned}
$$

Hence, by using Gronwall's inequality, we obtain that there exists a positive constant $C>0$ such that for every $\varepsilon>0, t \in[0, T]$,

$$
\begin{aligned}
\left\|\mathbb{F}_{t}^{\varepsilon}\left[\begin{array}{l}
v_{0} \\
y_{0}
\end{array}\right]-\mathbb{F}_{t}^{0}\left[\begin{array}{l}
v_{0} \\
y_{0}
\end{array}\right]\right\|_{X} \leqslant & C\left(\sup _{t \in[0, T]}\left\|\mathbb{T}_{t}^{\varepsilon}\left[\begin{array}{l}
v_{0} \\
y_{0}
\end{array}\right]-\mathbb{T}_{t}^{0}\left[\begin{array}{l}
v_{0} \\
y_{0}
\end{array}\right]\right\|_{X}\right. \\
& +\int_{0}^{T}\left\|\Pi_{\varepsilon} \mathbb{F}_{\eta}^{0}\left[\begin{array}{l}
v_{0} \\
y_{0}
\end{array}\right]-\Pi_{\eta}^{0} \mathbb{F}_{\eta}^{0}\left[\begin{array}{c}
v_{0} \\
y_{0}
\end{array}\right]\right\|_{X} \mathrm{~d} \eta \\
& \left.+\int_{0}^{T} \sup _{t \in[0, T]}\left\|\mathbb{T}_{t-\eta}^{\varepsilon}\left(-B B_{0}^{*} \Pi_{0} \mathbb{F}_{\eta}^{0}\right)\left[\begin{array}{c}
v_{0} \\
y_{0}
\end{array}\right]-\mathbb{T}_{t-\eta}^{0}\left(-B_{0} B_{0}^{*} \Pi_{0} \mathbb{F}_{\eta}^{0}\right)\left[\begin{array}{c}
v_{0} \\
y_{0}
\end{array}\right]\right\|_{X} \mathrm{~d} \eta\right) \exp (C T) .
\end{aligned}
$$

By using Proposition 4.2, the convergence of the Riccati operators i.e. (3.6) and Proposition 4.5 we see that the three terms in right hand side of the above formula converge to 0 as $\varepsilon \rightarrow 0$. Then, we immediately deduce (3.7).

Finally, we obtain the convergence of the optimal controls (3.8), using the definition of optimal controls in function of the closed-loop semigroup, i.e. (2.17), and the convergence of the closed-loop semigroup, i.e. (3.7).

\section{CONCLUding REMARKS}

In this paper we prove that the penalization of the free divergence condition allows the approximation infinite horizon LQR problem for the linearized Boussinesq system by the corresponding problems for a sequence of parabolic systems, for which standard theory and available computational techniques fully apply. The main open question left by this work is the study of the action of the obtained controllers when inserted in the nonlinear Boussinesq system. As in the case of the Navier-Stokes equation (see, for instance, [24]), we expect local stabilization results, the difficulty being that we need estimates for the nonlinear problem which are independent of $\varepsilon$.

We end this paper by formulating some comments and some open questions related to our main results in Theorem 3.1 and Theorem 3.2. 
- In Theorem 3.2, we can actually replace (3.9) by

$$
B u=\left[\begin{array}{c}
u_{1} \\
\vdots \\
u_{d-1} \\
0 \\
u_{d+1}
\end{array}\right] \mathbb{1}_{\mathcal{O}} \quad(u \in U)
$$

that is to say, a control operator that acts only on the first $(d-1)$ velocity components and on the temperature, see Remark 5.9 above.

- In the spirit of Theorem 4.8 of [4], assuming that (3.4) and (3.5) hold true, we may wonder if we have the following convergence in norm operator

$$
\lim _{\varepsilon \rightarrow 0+}\left\|\Pi_{\varepsilon}-\Pi_{0}\right\|_{\mathcal{L}(H ; X)}=0
$$

- For numerical purposes, it would be relevant to obtain error estimates for the convergences (3.6), (3.7) and (3.8), in the spirit of [20].

- A natural generalization, concerning both Theorem 3.1 and Theorem 3.2, could consist in adding an observation operator in the quadratic functionals defined in (1.7) and (1.8). We think that, at least in the case of bounded observation operators, our approach can be adapted to this situation, in the spirit of what has been done in [4] for Galerkin type approximations.

- For proving Theorem 3.1 and Theorem 3.2, the approach that we follow crucially uses the fact that $B$ and $B_{0}$ are bounded control operators. It would be relevant to extend these results for unbounded control operators in order to treat boundary control operators, see [2] for finite time horizon problem in the context of the Oseen system.

- Another important issue, namely when one aims getting closer to applications, is to show that if (3.3) or (3.9) holds then the semigroup generated by $A-B_{0} B_{0}^{*} \Pi^{\varepsilon}$ is uniformly exponentially stable for $\varepsilon$ sufficiently small.

- In the spirit of [3], it would also be interesting to obtain local exponential stabilization with a finite number of controls, uniformly with respect to $\varepsilon$, for the (nonlinear) Boussinesq system (1.1), replacing $\operatorname{div} v=0$ by $\operatorname{div} v+\varepsilon p=0$.

Acknowledgements. This study has been carried out with financial support from the French State, managed by the French National Research Agency (ANR) in the frame of the "Investments for the future" Programme IdEx Bordeaux - SysNum (ANR-10-IDEX-03-02). This work has also been supported by the grant "Numerical simulation and optimal control in view of temperature regulation in smart buildings" of the Nouvelle Aquitaine Region. The authors would like to deeply thank Mejdi Azaiez for many useful discussions and comments on this work.

\section{REFERENCES}

[1] M. Badra, Global Carleman inequalities for Stokes and penalized Stokes equations. Math. Control Relat. Fields 1 (2011) $149-175$.

[2] M. Badra, J.-M. Buchot and L. Thevenet, Méthode de pénalisation pour le contrôle frontière des équations de Navier-Stokes. J. Européen des Systèmes Automatisés (JESA) 45 (2012) 595-630.

[3] M. Badra and T. Takahashi, Stabilization of parabolic nonlinear systems with finite dimensional feedback or dynamical controllers: application to the Navier-Stokes system. SIAM J. Control Optim. 49 (2011) 420-463.

[4] H.T. Banks and K. Ito, Approximation in LQR problems for infinite-dimensional systems with unbounded input operators. $J$. Math. Syst. Estim. Control 7 (1997) 1-34.

[5] H.T. Banks and K. Kunisch, The linear regulator problem for parabolic systems. SIAM J. Control Optim. 22 (1984) 684-698.

[6] J.A. Bárcena-Petisco, Null controllability of a penalized stokes problem in dimension two with one scalar control. Asymptotic Anal. (2020) 1-38. 
[7] J.A. Bárcena-Petisco and K. Le Balc'h, Local null controllability of the penalized Boussinesq system with a reduced number of controls (2020). Preprint https://hal.archives-ouvertes.fr/hal-02913358.

[8] P. Benner, M. Heinkenschloss, J. Saak and H.K. Weichelt, Efficient solution of large-scale algebraic Riccati equations associated with index-2 daes via the inexact low-rank Newton-Adi method. Appl. Numer. Math. 152 (2020) 338-354.

[9] J. Borggaard, J.A. Burns, A. Surana and L. Zietsman, Control, estimation and optimization of energy efficient buildings. In 2009 American Control Conference, IEEE (2009) 837-841.

[10] J.A. Burns, X. He and W. Hu, Feedback stabilization of a thermal fluid system with mixed boundary control. Comput. Math. Appl. 71 (2016) 2170-2191.

[11] J.-M. Coron, Control and nonlinearity, vol. 136 of Mathematical Surveys and Monographs. American Mathematical Society, Providence, RI (2007).

[12] R.F. Curtain and G. Weiss, Well posedness of triples of operators (in the sense of linear systems theory), in Control and estimation of distributed parameter systems (Vorau, 1988). In Vol. 91 of Internat. Ser. Numer. Math. Birkhäuser, Basel (1989) 41-59.

[13] E. Fernández-Cara and S. Guerrero, Global carleman inequalities for parabolic systems and applications to controllability. SIAM J. Control Optim. 45 (2006) 1395-1446.

[14] A.V. Fursikov and O.Y. Imanuvilov, Controllability of evolution equations. Vol. 34 of Lecture Notes Series. Seoul National University, Research Institute of Mathematics, Global Analysis Research Center, Seoul (1996).

[15] J.S. Gibson, The Riccati integral equations for optimal control problems on Hilbert spaces. SIAM J. Control Optim. 17 (1979) $537-565$.

[16] P. Grabowski and F.M. Callier, Admissible observation operators. Semigroup criteria of admissibility. Integr. Equ. Oper. Theory 25 (1996) 182-198.

[17] F.-K. Hebeker, W. Wendland and M. Crouzeix, The penalty method applied to the instationary Stokes equations. Appl. Anal. 14 (1982) 137-154.

[18] M. Heinkenschloss, D.C. Sorensen and K. Sun, Balanced truncation model reduction for a class of descriptor systems with application to the Oseen equations. SIAM J. Sci. Comput. 30 (2008) 1038-1063.

[19] O.Y. Imanuvilov, J.P. Puel and M. Yamamoto, Carleman estimates for parabolic equations with nonhomogeneous boundary conditions. Chin. Ann. Math. Ser. B 30 (2009) 333-378.

[20] M. Kroller and K. Kunisch, Convergence rates for the feedback operators arising in the linear quadratic regulator problem governed by parabolic equations. SIAM J. Numer. Anal. 28 (1991) 1350-1385.

[21] E.-M. Ouhabaz, Analysis of heat equations on domains. In Vol. 31 of London Mathematical Society Monographs. Princeton University Press (2009).

[22] A. Pazy, Semigroups of linear operators and applications to partial differential equations. Vol. 44 of Applied Mathematical Sciences. Springer-Verlag, New York (1983).

[23] M. Ramaswamy, J.-P. Raymond and A. Roy, Boundary feedback stabilization of the Boussinesq system with mixed boundary conditions. J. Differ. Equ. 266 (2019) 4268-4304.

[24] J.-P. Raymond, Feedback boundary stabilization of the three-dimensional incompressible Navier-Stokes equations. J. Math. Pures Appl. 87 (2007) 627-669.

[25] J.-P. Raymond, Stokes and Navier-Stokes equations with nonhomogeneous boundary conditions. Ann. Inst. Henri Poincaré Anal. Non Linéaire 24 (2007) 921-951.

[26] T. Reis, Controllability and observability of infinite-dimensional descriptor systems. IEEE Trans. Autom. Control 53 (2008) 929-940.

[27] J. Shen, On error estimates of projection methods for Navier-Stokes equations: first-order schemes. SIAM J. Numer. Anal. 29 (1992) 57-77.

[28] J. Shen, On error estimates of some higher order projection and penalty-projection methods for Navier-Stokes equations. Numer. Math. 62 (1992) 49-73.

[29] H. Sohr, The Navier-Stokes equations. An elementary functional analytic approach. Birkhäuser Advanced Texts: Basler Lehrbücher, Birkhäuser Verlag, Basel (2001).

[30] O. Staffans and G. Weiss, Transfer functions of regular linear systems part ii: the system operator and the Lax-Phillips semigroup. Trans. Am. Math. Soc. 354 (2002) 3229-3262.

[31] R. Temam, Une méthode d'approximation de la solution des équations de Navier-Stokes. Bull. Soc. Math. France 96 (1968) $115-152$.

[32] M. Tucsnak and G. Weiss, Observation and Control for Operator Semigroups. Birkhäuser Verlag, Basel (2009).

[33] U. Vaidya, R. Rajaram and S. Dasgupta, Actuator and sensor placement in linear advection PDE with building system application. J. Math. Anal. Appl. 394 (2012) 213-224. 